\title{
A systems view of epithelial-mesenchymal transition signaling states
}

\author{
Stuart Thomson · Filippo Petti • Izabela Sujka-Kwok • \\ Peter Mercado - James Bean - Melissa Monaghan - Sean L. Seymour • \\ Gretchen M. Argast · David M. Epstein · John D. Haley
}

Received: 22 September 2010/Accepted: 7 December 2010/Published online: 31 December 2010

(C) The Author(s) 2010. This article is published with open access at Springerlink.com

\begin{abstract}
Abtract Epithelial-mesenchymal transition (EMT) is an important contributor to the invasion and metastasis of epithelial-derived cancers. While considerable effort has focused in the regulators involved in the transition process, we have focused on consequences of EMT to prosurvival signaling. Changes in distinct metastable and 'epigentically-fixed' EMT states were measured by correlation of protein, phosphoprotein, phosphopeptide and RNA transcript abundance. The assembly of 1167 modulated components into functional systems or machines simplified biological understanding and increased prediction confidence highlighting four functional groups: cell adhesion and migration, metabolism, transcription nodes and proliferation/survival networks. A coordinate metabolic reduction in a cluster of 17 free-radical stress pathway components was observed and correlated with reduced glycolytic and increased oxidative phosphorylation enzyme capacity, consistent with reduced cell cycling and reduced need for macromolecular biosynthesis in the mesenchymal state. An attenuation of EGFR autophosphorylation and a switch from autocrine to paracrine-competent EGFR signaling was implicated in the enablement of tumor cell chemotaxis. A similar attenuation of IGF1R, MET and
\end{abstract}

Electronic supplementary material The online version of this article (doi:10.1007/s10585-010-9367-3) contains supplementary material, which is available to authorized users.

S. Thomson - F. Petti - I. Sujka-Kwok · P. Mercado - J. Bean · M. Monaghan - G. M. Argast · D. M. Epstein - J. D. Haley ( $\square)$ Translational Research, OSI Pharmaceuticals Inc, 1 Bioscience Park Drive, Farmingdale, NY 11735, USA

e-mail: jhaley@osip.com

S. L. Seymour

AB Sciex, Foster City, CA 94404, USA
RON signaling with EMT was observed. In contrast, EMT increased prosurvival autocrine IL11/IL6-JAK2-STAT signaling, autocrine fibronectin-integrin $\alpha 5 \beta 1$ activation, autocrine Axl/Tyro3/PDGFR/FGFR RTK signaling and autocrine TGF $\beta$ R signaling. A relatively uniform loss of polarity and cell-cell junction linkages to actin cytoskeleton and intermediate filaments was measured at a systems level. A more heterogeneous gain of ECM remodeling and associated with invasion and migration was observed. Correlation to stem cell, EMT, invasion and metastasis datasets revealed the greatest similarity with normal and cancerous breast stem cell populations, CD $49 \mathrm{f}^{\mathrm{hi}} / \mathrm{EpCAM}^{-/ \mathrm{lo}}$ and $\mathrm{CD} 44^{\mathrm{hi}} / \mathrm{CD} 24^{\mathrm{lo}}$, respectively.

Keywords Cell polarity $\cdot$ Drug resistance $\cdot$ Epithelial mesenchymal transition - EMT · Non-small cell lung cancer · Phosphorylation - Snail - Systems biology ·

TGF beta $\cdot$ Zeb1 $\cdot$ Kinase

$\begin{array}{ll}\text { Abbreviations } \\ \text { NSCLC } & \text { Non-small cell lung carcinoma } \\ \text { pY } & \text { Phosphotyrosine } \\ \text { pS } & \text { Phosphoserine } \\ \text { pT } & \text { Phosphothreonine } \\ \text { SCX } & \text { Strong cation exchange chromatography } \\ \text { C18 } & \text { Octadecyl silica reverse-phase chromatography } \\ \text { LC } & \text { Liquid chromatography } \\ \text { EMT } & \text { Epithelial mesenchymal transition }\end{array}$

Introduction

Epithelial-mesenchymal-like transitions (EMT) are important in the progression of human carcinomas to a more 
invasive, metastatic capacity. While much effort has focused on the regulators and processes involved in the conversion of cells from an epithelial to mesenchymal state, relatively less effort has been invested toward the consequences of EMT on tumor cell resistance to chemo-, radioand targeted-therapies. Using models of both metastable and epigenetically-fixed EMT, we have sought to define at a molecular level the signaling and survival changes which occur as a consequence of EMT.

Epithelial-derived tumor cells are capable of transdifferentiation to a more mesenchymal phenotype, a process resembling EMT [1, 2]. Similarly epithelial tumors may, directly or through EMT, promote genetic alterations, a loss of heterozygosity and a wound-like activation of surrounding or EMT-generated stromal cells [3, 4]. Tumor localized inflammatory cells and stromal fibroblasts can comprise up to $50-70 \%$ of the tumor mass and there is active multi-directional communication between these co-evolving [5] cell types that comprise cancer tissue $[6,7]$. For example, tumor cells and infiltrating inflammatory cells promote the formation of desmoplastic stroma that provide paracrine survival factors to tumor cells [8] where tumor cells and cancer-associated fibroblasts become more similar than non-involved fibroblasts. Inflammatory signals in turn can promote EMT [9]. The activation and hyperproliferation of stromal cells and M2 macrophages facilitate migration of EMT-derived mesenchymal-like tumor cells [7, 10, 11]. Mesenchymal-like tumor cells gain migratory capacity at the expense of proliferative potential [12]. The migration and invasion of epithelial-derived carcinoma cells through basement membrane and into adjacent tissue has been associated with EMT-like mechanisms [13, 14], acting on single cells and/or cell sheets [15]. A reverse conversion, a mesenchymal-epithelial transition (MET), is thought to be required to regenerate a proliferative state and form macrometastases resembling the primary tumor at distant sites [16], though co-migration of EMT and non-EMT cells has been proposed [17]. There is histological evidence from patient tumor specimens suggesting that over time select tumor cells can co-express both proliferative and migratory programs and exhibit a spindleoid sarcoma-like phenotype [18]. While pure spindle-cell carcinomas of the lung are relatively infrequent, representing approximately two percent of NSCLC cases, sarcomatoid elements within tumor nests are more common and appear to be derived by EMTlike transitions [19].

The molecular circuitry that controls epithelial-mesenchymal transitions in normal embryonic development, in fibrotic diseases and wound repair, and during tumor progression has been an area of active investigation. The tumor heterogeneity, as a consequence of EMT and the relative insensitivity of mesenchymal-like tumor cells to targeted-, chemo- and radio- therapies, are important factors which determine overall patient outcome. Most notably, tumor cells deriving from EMT-like transitions have many of the properties of cancer stem cells [20] and show general resistance to chemotherapy [21, 22]. For example the EMT phenotype produced by Twist expression has been shown to inactivate pro-senescence pathways [23], however in general the molecular mechanisms promoting the anti-apoptotic phenotype associated with EMT are not well described at a molecular level.

Epithelial tissues normally use autocrine and paracrine ligand activation of EGFR family and IGF1R signaling to promote basal cell self-renewal, while in epithelial-derived cancers abnormal activation of these pathways promotes tumor expansion and progression. Our previous work has shown that through EMT-like transitions many carcinoma cell types acquire abnormal EGFR- and IGF1R-independent survival signals to escape anoikis and senescence during cell detachment and migration [24, 25]. EMTderived cells show relative resistance to selective IGF1R/ insulin receptor inhibition [24] or to EGFR inhibition [26-33] in multiple solid tumor types. This is paradoxical in that chronic activation of EGFR [34, 35] and IGF1R [36] can promote EMT-like transitions, but once having occurred these signals are rendered redundant and alternative pathways are engaged in the maintenance of the mesenchymal-like state. EMT-derived changes in survival, and metastatic signaling networks has medical importance in the development of anti-cancer combinations which not only cause apoptosis of primary tumor cells but also impact the migratory stem-like cell populations from which cancer can recur $[6,37,38]$.

Here we have investigated both the molecular changes which initially promote EMT-like transition as well as the more distant consequences toward survival, metabolic and cell adhesion signaling networks. At least three distinct EMT states can be discerned; a pre- or potential-EMT state, a reversible 'metastable' EMT state and an 'epigeneticallyfixed' EMT state. EMT-associated networks were identified and measured within three inducible 'metastable' models, H358/doxZeb1, H358/doxSnail and H358/TGF $\beta$. Similarly we compared network changes in four NSCLC lines with either epithelial or 'epigenetically-fixed' mesenchymal-like cell phenotypes. A combination of overlapping experimental approaches, measuring protein, phosphoprotein and RNA transcript abundance, were used to quantitate signaling network differences between these three different epithelial, metastable mesenchymal and epigenetically-fixed mesenchymal EMT states. The extensive cellular reprogramming associated with EMT resulted in marked shifts in autocrine survival, cell-junction and metabolic networks, relevant to drug discovery targeting EMT-like transitions and mesenchymal stem-like cancer cells. 


\section{Experimental procedures}

Full, detailed experimental and analysis procedures are provided in Supplemental Information.

Cell culture conditions, doxycycline-inducible EMT and immunoblotting

Human NSCLC lines H292, H358, Calu6 and H1703 were cultured in Dulbecco's modified Eagle's medium (DMEM) with $10 \%$ fetal bovine serum (FBS). To promote EMT in $\mathrm{H} 358$ cells TGF $\beta(10 \mathrm{ng} / \mathrm{ml})$ was added to complete media for 7 days. H358/Snail and H358/Zeb1 were expressed under the control of the doxycycline inducible CMV promoter (pTRE2puro; invitrogen.com) using the two vector TET repressor/TET activator system (ptTS, prTA; bla ${ }^{\mathrm{R}}$ ) based on [39]. Doxycycline $(0.5 \mu \mathrm{g} / \mathrm{ml})$ induction of transgene expression was verified by immunoblot. Receptor phosphotyrosine content in EMT model cell lines also was measured by blot e.l.i.s.a. (RTK array; rdsystems.com) under conditions detailed by the manufacturer.

\section{Confocal microscopy}

Cells grown on glass coverslips were washed and fixed with 4\% paraformaldehyde in Dulbecco's PBS followed by permeabilization in $0.1 \%$ Triton X-100. E-cadherin (Santa Cruz, sc21791) and vimentin (Chemicon, AB5733) primary antibodies overnight at room temperature and with diluted Alexa-fluor 488 or Alexa-fluor 568-conjugated secondary antibody for $1 \mathrm{~h}$. Nuclei were stained with 4',6-diamidino2-phenylindole ( $300 \mathrm{nmol} / \mathrm{l}$ for $5 \mathrm{~min}$ ). The images were captured with AxioVision software on a Zeiss AxioObserver microscope and camera and Apotome accessory.

\section{RNA isolation, DNA microarray and RT-PCR analysis}

RNA isolated from H358/doxZeb1, H358/doxSnail, H358/ TGF $\beta$ or parental $\mathrm{H} 358$ cells was reverse transcribed into labeled cDNA and hybridized to U133 Plus 2 or Hu1.0 exon arrays under conditions described by the manufacturer (affymetrix.com).

Two fold RNA abundance differences with detection $P$ values of $<0.05$ in any of the three H358 models were used as a cutoff. RT-PCR was performed under standard conditions to generate $\Delta \Delta \mathrm{Ct}$ values for control normalized mesenchymal—epithelial RNA transcript abundance ratios.

Preparation of cell extracts and phosphorylation directed affinity chromatography

Membrane, cytosol and nuclear protein fractions were prepared, denatured in $8 \mathrm{M}$ urea, reduced, alkylated, subjected to proteolysis with trypsin and desalted on C18 resin.
Anti-phosphotyrosine affinity fractions were isolated from Triton X-100 cell lysates as previously described [40, 41]. Serine and threonine phosphorylated peptides were enriched by binding to titanium dioxide. Essentially, phosphopeptides were enriched on $\mathrm{TiO}_{2}$ beads under acidic conditions and eluted at high $\mathrm{pH}, 50 \%$ acetonitrile. Lyophilized $\mathrm{TiO}_{2}$ bound and flow-through fractions were derivitized with stable isotope tags, isotopically labeled peptides combined and subjected to SCX ion exchange, C18 step chromatography, followed LC-tandemMS. Relative peptide quantitation was performed by stable isotope peptide derivitization [42].

Peptide identification and quantification by LC-tandem MS

Tandem mass spectrometry was performed as previously described [40]. Proteins were identified from survey and product ion spectra using the Paragon algorithm of ProteinPilot (absciex.com) searching human SwissProt and UniProt protein databases. When multiple protein isoforms were identified, only peptides specific to each detected isoform were used, which factored in ion counts for weighting in the protein ratio calculation [43]. Parsimony of protein results was assured by rigorous protein inference with the ProGroup algorithm. Protein identification complied with the guidelines of [44] where 2 or more unique isoform-specific peptides were required for inclusion. Proteins identified with $\geq 95 \%$ confidence with relative abundances between experimental conditions in the upper and lower distribution quartiles $(>75 \%$ or $<25 \%)$ and with a $t$-test $P$ value (for any difference between cell line or biological condition) of $<0.05$ were further considered. Under these conditions false discovery rates (FDR) of multiple datasets were typically $<1 \%$ and correctly binned previously defined benchmark proteins, E-cadherin, $\gamma$-catenin, $\alpha$-catenin, vimentin and fibronectin to their respective epithelial or mesenchymal cell states. After protein identification at $95 \%$ confidence with 2 or more unique peptides, at least two independent lines of evidence were required for further consideration.

Phosphoserine and phosphothreonine containing peptides were identified from TGF $\beta$ induced $\mathrm{H} 358$ cells ( 1 day after TGF $\beta$ treatment, $10 \mathrm{ng} / \mathrm{ml}$ ) using ProteinPilot v3.0 (absciex.com) and a 95\% peptide confidence threshold. Phosphopeptides were measured by determination of the median normalized iTRAQ quant tag peak areas for the biological duplicate samples. EMT modulated phosphopeptides were selected where the TGF $\beta /$ control $\log 2$ ratio was $>1$ or $<-1$ and where the control N1/control N2 was between $\log 2-0.5$ and 0.5. (Supplemental Tables S9 and S10). 
Functional analysis and performance parameters

A schema for the statistical and categorical analysis of protein, phosphopeptide and RNA transcript data is provided in Supplemental Fig. S1. Changes in the abundance of proteins, phosphopeptides and RNA transcripts were compared between fractions, experiments and models where membership within specific signaling networks only was established using two or more independent lines of evidence (Supplementary Tables S4-S8). Pair wise and three way comparisons (Snail-Zeb1 overlap, Snail-TGF $\beta$ overlap, TGF $\beta$-Zeb1 overlap and Snail-TGF $\beta$-Zeb1 overlaps, as well as Calu6 and H1703 overlaps) are presented in Supplementary Table S3. Cellular components modulated through EMT (942 components) specifically in a single model are listed in Supplementary Table 12. Cross-correlation between conditions and the assembly of proteins and encoded proteins within biological systems and assemblies were used to increase statistical significance and facilitate functional understanding. Proteins and RNA transcripts differentially expressed between EMT-like cell states were grouped according to function using current literature, DAVID, Ingenuity Pathway Assist (IPA) and STRING. GO terms were annotationally clustered using DAVID using a significance $P$ value cutoff of 0.05 (enrichment score $>1.3$ ) (Supplemental Table S2). In the functional illustrations of complexes modulated during EMT, proteins were assembled by protein-protein contacts and grouped by systems or machines.

\section{Results and Discussion}

Epithelial-mesenchymal transition (EMT) involves extensive transcriptional reprogramming [45] to partially or fully disassemble epithelial cell sheets and promote the invasion, migration and survival of disseminated mesenchymal-like tumor cells. In order to understand the biological consequences of these different states of EMT upon cancer cells we initiated studies to measure the changes in cellular signaling, particularly those pathways which promote cell survival and resistance to anti-cancer therapies, in models that represent these different states of EMT. To achieve this we created three models in which EMT was inducible and isogenic to the NSCLC H358 cell line, which harbors a transforming mutation in K-ras, exhibits an epithelial phenotype and forms cell-cell junctions. H358 cells stably transfected with doxycycline-inducible Zeb1 or Snail cDNAs or exposed to exogenous TGF $\beta(10 \mathrm{nM})$ over a 7-day period promoted the downregulation of the epithelial cell marker E-cadherin and the upregulation of the mesenchymal cell markers vimentin and fibronectin, characteristic of an EMT-like transition. Changes were measured in an isogenic manner, in the presence and absence of the EMT inducers Zeb1, Snail and TGF $\beta$. Thirty-five individual clones expressing Zeb1 and 23 clones expressing Snail were isolated and characterized for transgene induction and the expression of EMT markers E-cadherin, $\gamma$-catenin, vimentin, fibronectin, with increasing concentrations of doxycycline added to the culture media (Supplementary Figs. S2 and S3). As a control, three vector only clones were isolated and characterized in the presence and absence of doxycycline. No change in EMT markers was observed in the vector control lines with doycycline treatment, either by Western blot, by proteomic LC-MS/MS analysis or by microarray analysis (Supplementary Fig. S4). We also compared these inducible models (H358/doxZeb1, H358/ doxSnail, H358/TGF $\beta$ and H358/vector control) to four phenotypically epithelial or mesenchymal-like NSCLC lines (H292, H358, Calu6 and H1703). The phenotypes of the epithelial and mesenchymal-like tumor models were characterized by immunofluorescence microscopy examining co-staining for E-cadherin and vimentin and by light microscopy as shown in Fig. 1a. The epithelial models form cell-cell junctions and express membrane localized E-cadherin. In contrast cell models with a mesenchymal phenotype (Calu6, H1703, H358/TGF $\beta$, H358/Snail and H358/ Zeb1) exhibited a characteristic scattered spindloid morphology (Fig. 1a and Supplementary Fig. S5), lacked E-cadherin expression and expressed perinuclear intermediate filaments containing vimentin (Fig. 1a) and low levels of cytokeratins 8 and 18 (not shown). These key protein changes between epithelial and mesenchymal NSCLC states served as an expected benchmark demonstrating EMT-like changes and allowed for further experiments measuring signaling network associated with EMT states.

The metastable state of these inducible models was reflected by the complete or partial reversion by removal of the EMT stimulus, for example by removal of $\operatorname{TGF} \beta$ or doxycycline from the inducible $\mathrm{H} 358$ models, which restores cell-cell junctions and their associations with the actin cytoskeleton [25]. Withdrawal of doxycycline from the induced Zeb1 and Snail models similarly reversed EMT component changes (Supplementary Fig. S8). Continuous EMT induction for up to 4 months did not appreciably alter the reversible metastable phenotype. A reversible plastic metastable state has been observed in vivo in cells expressing epithelial and mesenchymal splice forms of FGFR2 [46]. Treatment of the 'epigenetically-fixed' H1703 or Calu6 cells with 5-aza-2'-deoxycytidine (10 $\mathrm{nM}$ ) for 14 days resulted in decreased vimentin protein and increased E-cadherin and ErbB3 proteins (data not shown), consistent with mesenchymal to epithelial transition observed with exposure to HDAC inhibitors [32]. EMT appears to be a continuum, wherein three distinct states can be discerned; (1) a potentialEMT state where mesenchymal markers can be expressed in epithelial sheets with intact cell-junctions; (2) an early 

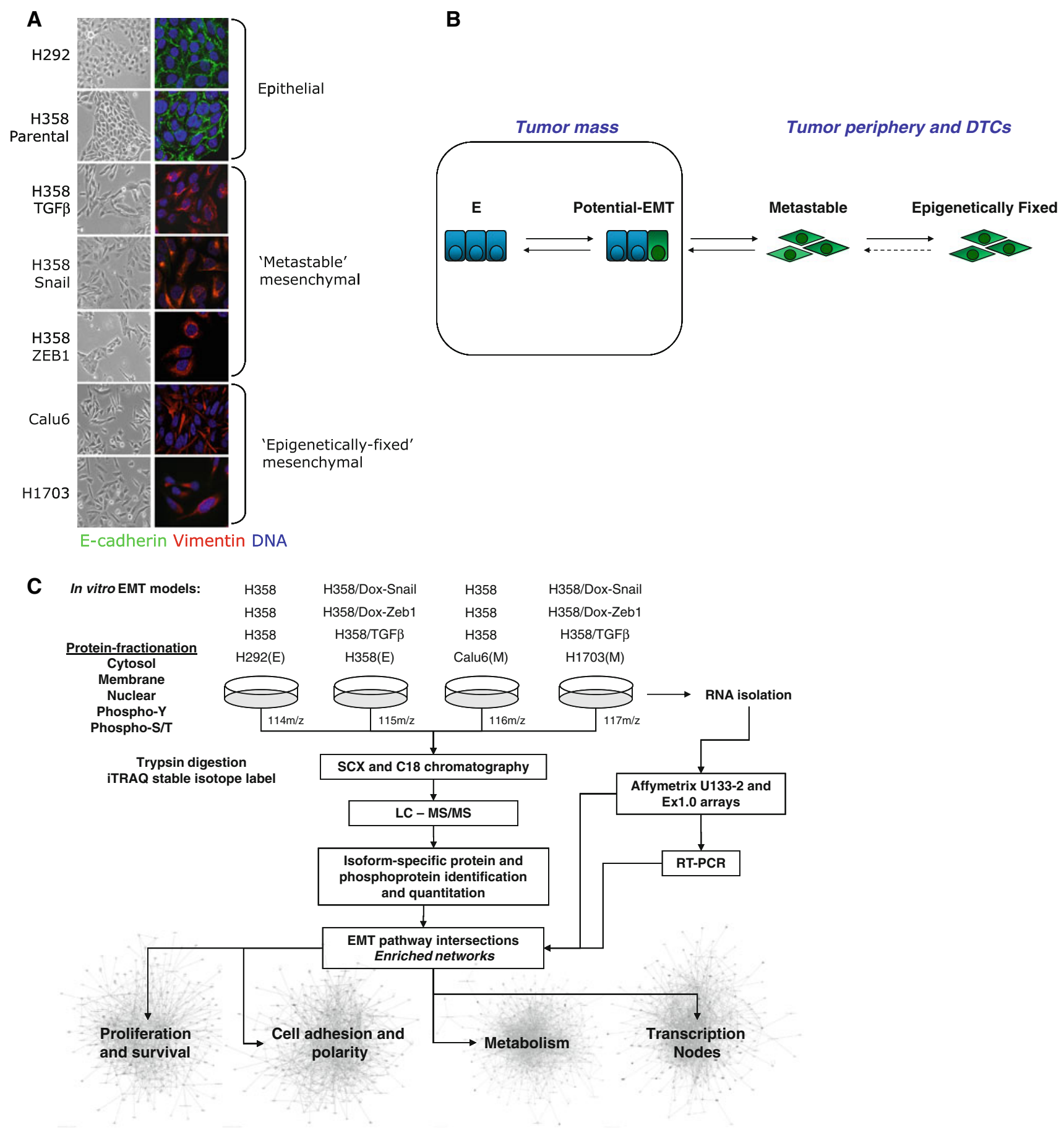

Fig. 1 a NSCLC model phenotypes for H292, H358, H358/TGF $\beta$, H358/doxSnail, H358/doxZeb1, Calu6 and H1703 showing phase contrast and immunofluorescence images using anti-E-cadherin, antivimentin and DAPI co-staining. E-cadherin containing cell junctions (green) are visible in the epithelial lines $\mathrm{H} 292$ and H358. In contrast the metastable H358/TGF $\beta$, H358/doxSnail and H358/doxZeb1 cells show a loss of E-cadherin expression, a gain of vimentin expression (red) and a characteristic scattered phenotype. Similarly the

reversible 'metastable' EMT state; and (3) an 'epigenetically-fixed' EMT state, irreversible except by chromatin remodeling $[32,47]$. Overall, the model systems used here epigentically fixed NSCLC lines Calu6 and H1703 lack E-cadherin containing cell-cell junctions and express perinuclear vimentin, an intermediate filament component. b A scheme depicting EMT states within the primary tumor mass, in the tumor periphery and in disseminated tumor cells (DTCs). c Experimental schema for cell fractionation, protein, phosphoprotein and RNA measurements and network enrichment

represent both epithelial and mesenchymal-like EMT phenotypes and metastable and epigenetically-fixed chromatin states (Fig. 1b). 
The consequences of EMT state on signaling changes associated with therapeutic resistance were measured by global measurement of protein, phosphoprotein and RNA transcript abundance (Fig. 1c). Component changes were cross-correlated between cellular fractions, experiments and models where membership within specific signaling networks was established using two or more independent lines of evidence. Using this stringent cross-correlation strategy, 218 proteins, 146 phosphoproteins and $\sim 1200$ RNA transcripts showed differential abundance in the multiple EMT models. Details and statistics are given in Supplementary Table S1. One hundred sixty-nine EMT components were confirmed by either RT-PCR, immunoblot or confocal IF (Supplementary Table S8). Further validation was obtained by comparison of the model systems to each other and to model systems (EMT, stem cell and metastatic; Supplementary Table S8) present within the literature, where few of the EMT components listed in Supplementary Tables S4-S8 were modulated in one model alone. Components specific to a given single model are listed in Supplementary Table S12. Biological functions were clustered and components perturbed by EMT assembled into systems to increase significance and facilitate functional understanding.

Metastable and epigenetically-fixed EMT states show molecular heterogeneity

Significantly enriched functional networks altered between epithelial and mesenchymal states were identified by functional annotation clustering [48, 49] using a significance threshold of $P<0.05$ (Fig. 2a; Supplementary Table S2). Highly enriched functions altered by EMT included (1) the marked alterations in cell-cell junction and adhesion networks; (2) the switching of cell proliferation and survival networks associated with therapeutic resistance; and (3) the transcriptional networks required for reprogramming cell adhesion, cell survival, redox stress and metabolic pathways. Hierarchical clustering and data intersection analysis of EMT components modulated by Zeb1, Snail and TGF $\beta$ revealed differences between the three EMT inducers. From a total of 2973 genes modulated in any of the three H358 EMT models, $16 \%$ were shared between Zeb1, Snail and TGF $\beta$ models, $13.2 \%$ were shared between Snail and Zeb1, while only $5.6 \%$ were shared between Snail or Zeb1 and the TGF $\beta$ induced EMT state (Supplementary Table S3), where the false discovery rate (FDR) was $<1 \%$. EMT components exclusively modulated in a single model, for example with Zeb1 but not with Snail or TGF $\beta$, are listed in Supplementary Table S12. We examined both down regulated and up regulated gene sets independently to ask whether loss and gain of RNA transcripts were similarly correlated across models, by calculating overlapping genes or proteins as a percentage of non-redundant group size (Fig. 2b; Supplementary Table S3). Zeb1-Snail overlapping down-regulated genes were 3 -fold greater represented than either TGF $\beta$-Zeb1 or TGF $\beta$-Snail overlapping genes; up-regulated genes showed a 4-fold greater similarity between Zeb1 and Snail compared with TGF $\beta$. The subset and clustering analysis of mRNA abundance data indicated an increased diversity of modulated genes in the TGF $\beta$ model relative to the more closely related Zeb1 and Snail models.

We asked whether the loss of epithelial components was more uniform than the acquisition of mesenchymal components. The concordance of decreased epithelial genes (20\%) in all three inducible H358 models was slightly higher than the overlap of increased mesenchymal genes (15\% or 1.3 -fold). Similarly, comparison of the fixed-state NSCLC models showed concordance of decreased genes between EMT states to be 1.4-fold higher than increased genes ( $52 \%$ vs. $36 \%$, respectively). When we compared the reversible 'metastable' H358 models with the 'epigenetically-fixed' NSCLC lines the trend for concordance of loss of epithelial markers (10\%) over the gain of mesenchymal markers $(2 \%)$ was 5 -fold. These data indicate that the loss of epithelial components during EMT showed greater uniformity than the gain of mesenchymal components. These data suggest that the enhanced diversity of mesenchymal-like tumor cell states could contribute to the emergence of therapeutically resistant cell populations. This hypothesis is explored further below in the context of the extensive reprogramming of cell survival networks mediated by EMT-like transitions.

There are a number of literature reports of gene signatures correlating EMT, metastatic and stem cell phenotypes. Comparison of the EMT dataset obtained here with eighteen datasets from the stem cell, invasion/metastasis and EMT fields [50-63] indicate the NSCLC EMT models were most closely related with normal and cancer-derived mammary stem cells (Fig. 2c; Supplementary Tables S8 and S11). The greatest intersection of EMT modulated protein and mRNA abundance, adjusted for dataset size, were observed for normal mammary stem cells selected for $\mathrm{CD} 49 \mathrm{f}^{+} / \mathrm{EpCAM}^{\text {low }}[63]$ and breast cancer stem cells selected for $\mathrm{CD}_{4} 4^{+} / \mathrm{CD} 24^{-}$[51]. These comparisons do highlight the heterogeneity of EMT and stem cell model systems and states, suggesting a high degree of plasticity which may contribute to resistance to single agent anticancer therapies.

Reduced cell cycling in metastable but not epigenically-fixed EMT states

The metastable EMT phenotype driven by Zeb1, Snail or TGF $\beta$ was accompanied by a decrease in the transcription 
A

Enrichment for functional systems decreased (top) or increased (bottom) with EMT $(p<0.05)$. Wedge area is equal to the annotation enrichment score (range $10.0-1.4$; where 1.3 (range $10.0-1.4$, where 1.3 represents a $p$ value of 0.05 ).
Enrichment scores, annotation group member counts and $p$ values are detailed in Supplementary Table S2.
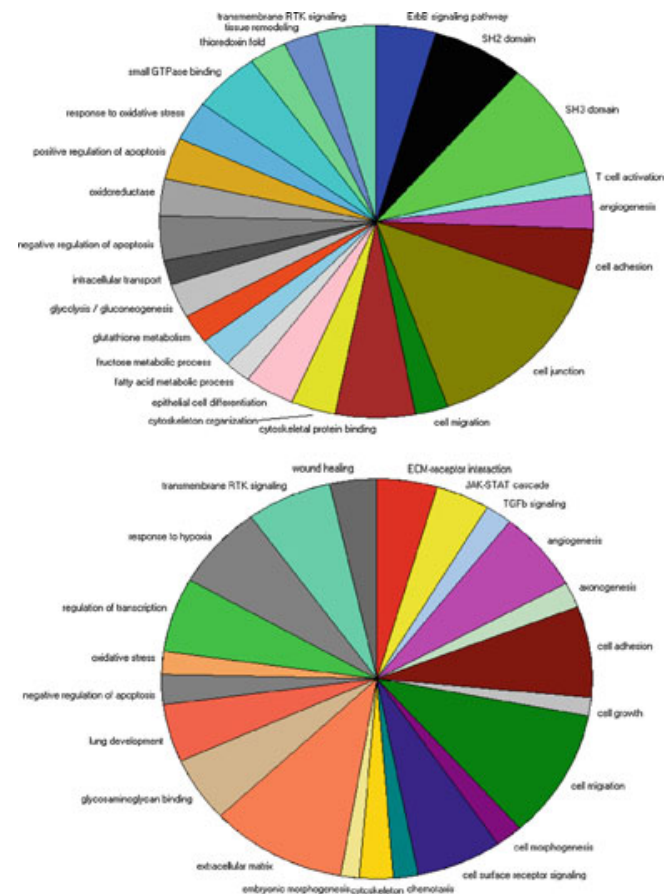

B

The up-regulation of EMT-induced mRNAs is more heterogeneous when compared to attenuated transcripts

\begin{tabular}{lllc}
\multicolumn{1}{c}{ Overlap } & \multicolumn{1}{c}{ Group Size } & \multicolumn{1}{c}{ Overlap } & \% Overlap \\
\hline \hline SNA-TGF Down & 1204 & 82 & $7 \%$ \\
SNA ZEB Down & 953 & 212 & $22 \%$ \\
TGF-ZEB Down & 1219 & 92 & $8 \%$ \\
SNA-TGF-ZEB Down & 1368 & 269 & $20 \%$ \\
\hline SNA TGF Up & 1089 & 58 & $5 \%$ \\
SNA-ZEB Up & 1026 & 251 & $24 \%$ \\
TGF-ZEB Up & 1126 & 67 & $6 \%$ \\
SNA-TGF-ZEB Up & 1341 & 200 & $15 \%$ \\
\hline H1703-CALU6 Down & 279 & 146 & $52 \%$ \\
H1703-CALU6 Up & 149 & 53 & $36 \%$ \\
H1703-CALU6-SNA-TGF-ZEB Down & 375 & 37 & $10 \%$ \\
H1703-CALU6-SNA-TGF-ZEB Up & 245 & 5 & $2 \%$ \\
\hline
\end{tabular}

C

EMT in NSCLC lines is most closely related to normal and cancer-derived mammary stem cells

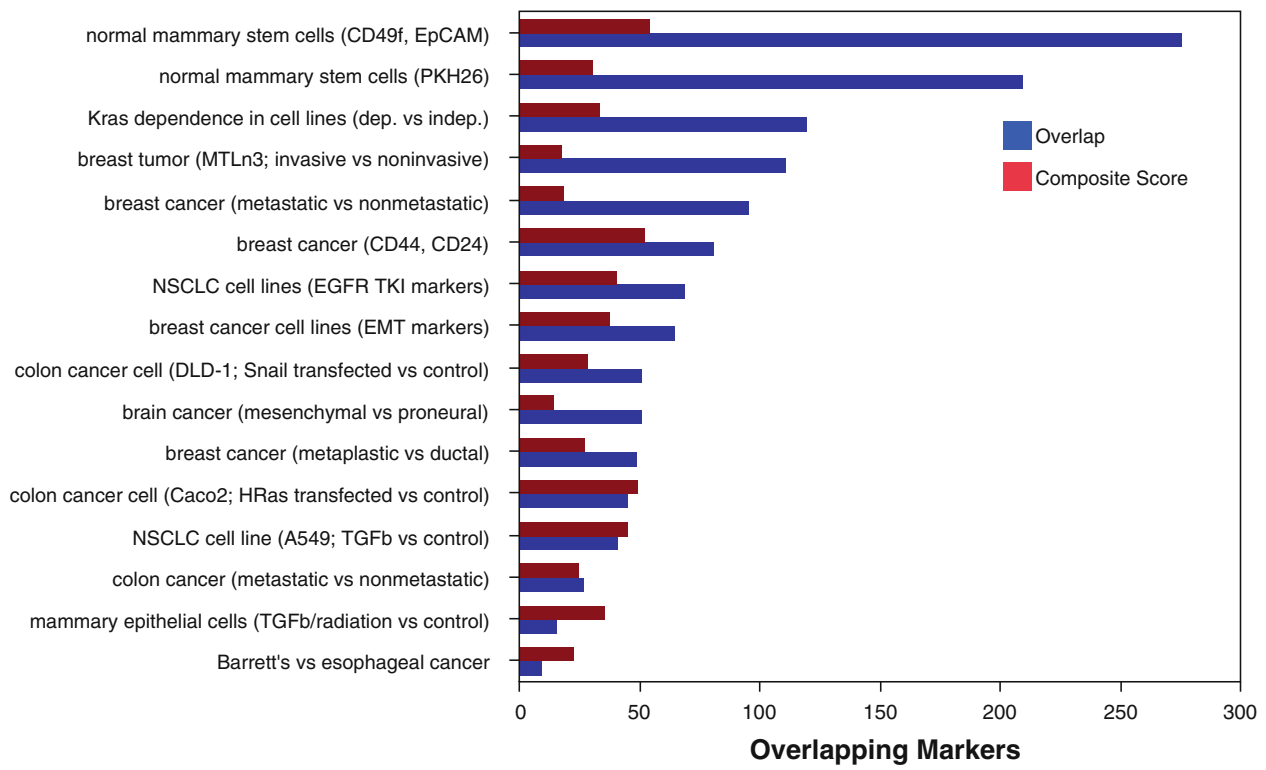

Fig. 2 a Enriched functional annotations from combined proteome and transcript profiles. b Heterogeneity of EMT states is in part dependent on the EMT inducer, where Zeb1- and Snail-induced EMT were more closely related than TGF $\beta$-induced EMT. c EMT dataset comparison to 18 datasets related stem cell, invasion/metastasis and

and regulatory phosphorylation of cell cycling components. These data were consistent with our previous findings that after 7 days TGF $\beta$ mediated EMT in H358 cells increased cell cycle time 1.7-fold over untreated control cells [64] where a general decrease in transcript levels for cyclin
EMT characteristics [50-63]. The NSCLC EMT dataset was most closely related to normal and cancer-derived mammary stem cells, either by number of overlapping markers or when adjusted for dataset size (composite score)

isoforms was observed (Supplementary Table S5). Reduced proliferation also was observed for the H358/ Zeb1 and H358/Snail models but not for the vector control H358 cells and not in the absence of doxycycline (Supplementary Fig. S6). Attenuated cell cycling with 
metastable EMT was apparent from phosphorylation site analysis early following TGF $\beta$ induced EMT (Supplementary Table S9). Within $24 \mathrm{~h}$ of TGF $\beta$ treatment decreased phosphorylation and activation of cell cycle kinases and their substrates was observed. For example, significantly reduced phosphorylation of DNA ligase 1 (LIG1) on S66 and S76, required for cell cycle traverse and DNA repair [65], MCM2 on S139, a site regulating cell cycle traverse and NUMA1 on S1757 and S1760 were observed. These data support the reduction in cell cycling in the H358 background within $24 \mathrm{~h}$ of EMT initiation by Zeb1, Snail and TGF $\beta$. This is in marked contrast to NSCLC epigenetically-fixed mesenchymal cells that show both the ability to migrate and proliferate. We have shown previously that there was no difference in proliferation rate, as measured by $\mathrm{BrdU}$ incorporation, when comparing epithelial and mesenchymal states [31].

Cell-junction assemblies and polarity networks are repressed with EMT

The loss of cell polarity and cell-cell junctions and the partial disassembly of epithelial tissues are changes associated with cancer progression to an invasive, metastatic state. The analyses presented here provide a comprehensive overview of the extensive changes observed in these important cellular protein complexes during the EMT process (Fig. 3). As expected [66] a marked and consistent down-regulation of cell-polarity, tight-junction, adherensjunction, desmosomal-junction and basement membrane adhesion components was correlated with a mesenchymal EMT state, events that served as a technical benchmark to validate the experimental and statistical approaches used. Note to verify these findings, representative protein and RNA changes were confirmed by immunoblot and TaqMan RT-PCR, respectively (Supplementary Table S4).

In both inducible H358 and established NSCLC model systems, a down regulation of thirty-three tight junction and polarity components was observed (Fig. 3). The organizing complex required for the establishment of apical-basal polarity in epithelial cells, comprising ParD3, ParD6, Crb3, Lin7C and LLGL2, was reduced in abundance following EMT, as was tyrosine phosphorylation of ParD3. Polarity organizing complexes with Par and $\mathrm{Crb}$ family proteins are required for the initiation of tight junction formation between adjacent epithelial cells [67]. Tight junctions [68] were assembled via the transmembrane receptors occludin (OCLN), claudin3 (CLDN3), claudin7 (CLDN7) and marvelD2 to link adjacent epithelial cells. These receptors in turn establish cell-cell mechanical strength by tethering to the actin cytoskeleton via ZO1/2/3 (TJPs), INADL, CGNL1, $\alpha$-catenin (CTNNA1) and eplin (LIMA1). The anchoring of claudin7 junctions to actin filaments also is thought to occur through its interaction with the epithelial marker Epcam (TACSTD1), $\alpha$-catenin and $\alpha$-actinin- 4 , which were markedly reduced in abundance in comparing mesenchymal and epithelial cell states. Similarly proteins involved in the assembly and linkage of junctional complexes with the actin cytoskeleton were attenuated with EMT, notably BAIAP2, DIAPH1 and AHNAK.

Twenty-two cell-cell adherens junction components were coordinately attenuated with EMT in the multiple model systems (Fig. 3). In adherens junctions [69], individual E-cadherin $(\mathrm{CDH} 1)$ and P-cadherin $(\mathrm{CDH} 3)$ proteins located on adjacent cells bind in a calcium-dependent manner and are linked to their respective actin cytoskeletons via $\beta$-catenin (CTNB1) $/ \gamma$-catenin (JUP), $\alpha$-catenin, eplin protein complex or through JUP, $\alpha$-catenin $(\mathrm{CTN})$ and eplin (LIMA1) linkages. Adherens junction assembly is regulated by protein tyrosine phosphorylation. Both total and tyrosine phosphorylated forms of $\alpha$-, $\beta$ - and $\delta$-catenins were attenuated in the fixed mesenchymal state, while metastable EMT models showed a more variable loss of adherens junction components 7 days post induction (Supplementary Table S4). Additional adherens junction linkages to the actin cytoskeleton are made through nectin2 (PVRL2), $\alpha$-catenin and eplin (LIMA1) or through afadin (MLLT4), LMO7 and $\alpha$-actinin4 (ACTN4), which were generally attenuated during EMT.

In desmosomal junctions, desmocollin2 (DSC2) and desmoglein2 (DCG2) on adjacent cells can form complexes with $\gamma$-catenin (JUP), $\alpha$-catenin and eplin (LIMA1) to link epithelial cells to the actin cytoskeleton [70]. DSG2 and DSC2 proteins are enriched in poorly differentiated basal epithelial cells. Linkage to $\gamma$-catenin, plakoglobin $2 / 3$ (PKP2/3) and desmoplakin (DSP) provide additional tethering to keratin $7,8,18$ and 19 containing intermediate filaments. These junction complex components were generally attenuated following EMT, with the exception of desmoglein, desmocollin and keratins in the single H358/ TGF $\beta$ model where no change or increased protein abundance was observed, respectively (Fig. 3, Supplementary Table S4). The loss of phosphotyrosine on cell junction components generally tracked with overall protein loss in the fixed state NSCLC models (adjusted $r^{2}=0.73$, $F<0.0001$ ), but was not significant in TGF $\beta$ induced H358 cells (adjusted $r^{2}=0.32, F<0.06$ ). Taken together these data indicate that, in the fixed-state models, protein loss, in part through reduced transcription, can account for much of the reduction in protein tyrosine phosphate content. Finally, the loss of $\alpha 6$ integrin, tetraspanin-1 (TSPAN1), laminin-A5 (LAMA5), ladinin (LAD1), AGR2/3 and MPZL2 (EVA) would be predicted to weaken cell-cell junctions and decrease cell attachment to basement membrane. 


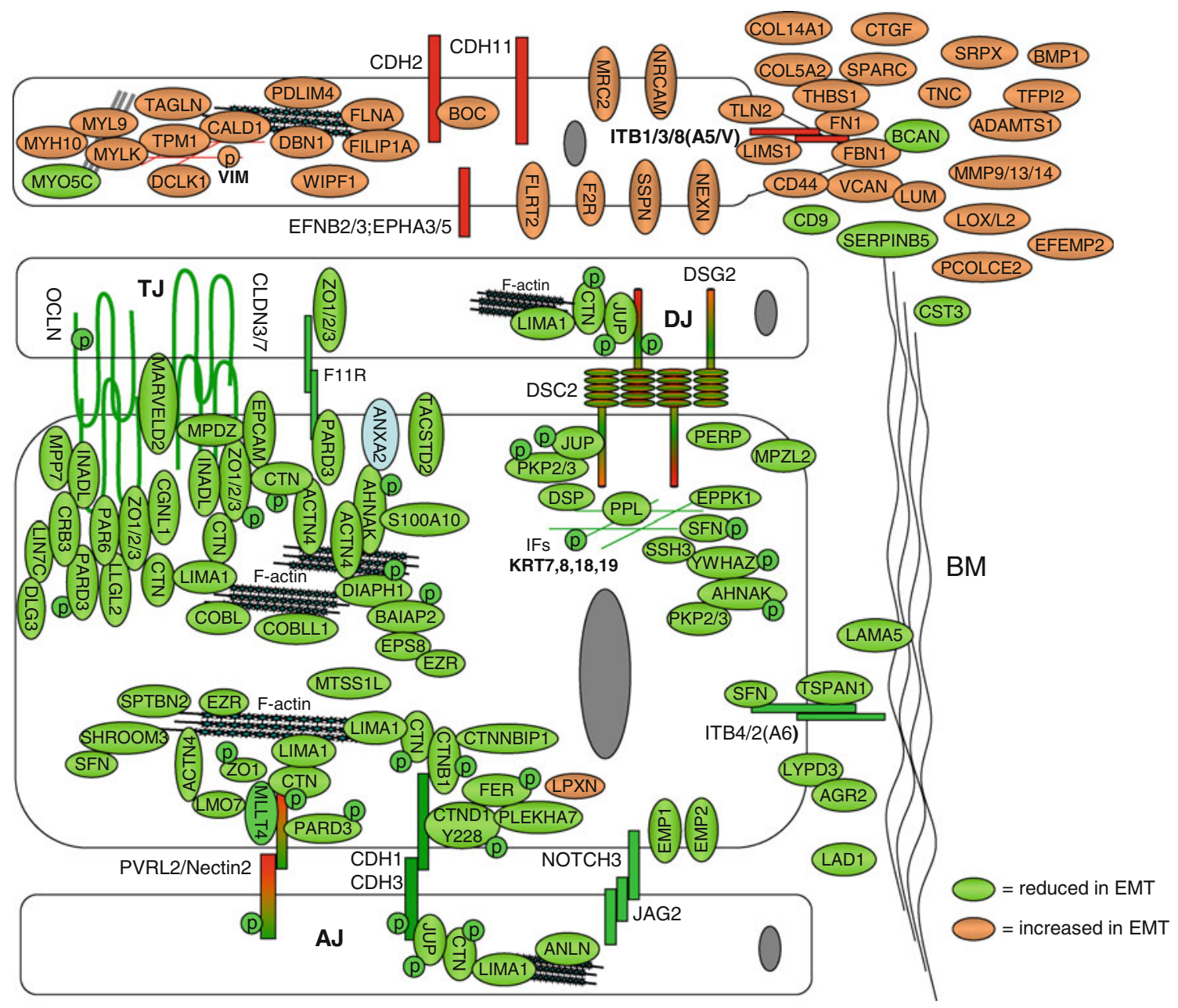

Fig. 3 EMT associated cell-cell junction and cell-adhesion/ECM changes, grouped by system and assembled by protein-protein contacts. Systems decreased or lost with EMT included tight junctions (TJ), adherens junctions (AJ), desmosomal junctions (DJ) and adhesion to basement membrane (BM). Systems increased with EMT included myosin motor coupling with intermediate filament and

Multiple cell junction components were differentially phosphorylated on serine, threonine or tyrosine early during the EMT process, $24 \mathrm{~h}$ after initiation of EMT by addition of exogenous TGF $\beta$. We performed phosphopeptide selection of iTRAQ labeled peptides by titanium dioxide $\left(\mathrm{TiO}_{2}\right)$ affinity selection followed by LC-tandem MS. Four hundred ninety-six individual phosphopeptides were identified using a 95\% confidence threshold (Supplementary Table S4). EMT modulation of phosphosite usage was assessed by Wilcoxon signed rank $(P<0.05)$. $\delta$-catenin (CTNND1) showed increased phosphorylation on S230, S349 and S352. The propeller protein AHNAK, known to participate in cell-cell junction formation through a complex with annexin A2, actin and S100A10, showed increased phosphorylation on S5448 and S5752, decreased phosphorylation of S5731. AHNAK homolog AHNAK2 was significantly increased on S857 and actin cytoskeletal assemblies, proteases responsible for BM degradation and extracellular matrix remodeling, and integrin/cadherin switching allowing new adhesion signals. Components in green were attenuated during EMT while those in light brown/red were increased with EMT

epiplakin (EPPK1) was increased on S3227. These data suggest the hypothesis that increased serine phosphorylation on these cytoskeletal/junction proteins, whose abundance will eventually be markedly decreased through EMT, may promote protein turnover and/or complex disassembly. The network approach used here highlights the marked attenuation of tight, adherens and desmosomal junction components, polarity organizing complexes and changes in adhesion signals in multiple EMT models.

Extracellular matrix remodeling and migratory motor systems acquired through EMT

We considered what new adhesive and cytoskeletal structures would compensate for the loss of epithelial cell-cell junctions, to mitigate anoikis. One such potential mechanism is through an increased dependence on intergin 
signaling. In the H358 EMT models integrin switching to $\alpha 5 \beta 1$ and autocrine fibronectin (FN) secretion was observed and activation of this integrin complex is consistent with increased tyrosine phosphorylation on PTK2/ FAK, paxillin and p130CAS (see below). In addition there are many changes to the content of the extracellular matrix surrounding tumor cells. For example, versican (VCAN), a matrix proteoglycan containing chondroitin sulfate acts to promote hyaluronan matrix assembly [71] and associates with lumican. Both versican and lumican were increased in the three metastable H358 models. Further markedly increased synthesis of extracellular matrix $(\mathrm{ECM})$ proteins collagen 14A1 and 5A2, tenacin C, THBS1, SPARC and fibronectin were noted.

The presence of basement membrane provides a stiff physical barrier which impedes tumor cell invasion. As part of the EMT process tumor cells increase the production of proteases that are able to degrade the surrounding basement membrane. The observed increased abundance of the metalloproteinases MMP14 (MT1), MMP9 and ADAMTS1 contribute to proteolytic cascades leading to basement membrane (BM) destruction and tumor cell invasion. In addition, multiple negative regulators of invasion and migration were coordinately down regulated, including CD9, BCAN and maspin (SERPINB5). Increased N-cadherin $(\mathrm{CDH} 2)$ and its associated protein BOC was observed together with cadherin-11 (CDH11), consistent with cadherin switching from E- to N-cadherin early in EMT [72]. Taken together these observations support the notion that as part of the genetic reprogramming during EMT tumor cells switch to a pro-invasive/migratory phenotype an essential component of the metastatic program.

In addition to these changes driving an invasive phenotype, numerous changes in proteins involved in cellular motility were observed. TGF $\beta$ and Snail mediated EMT in the H358 background were found to increase cell invasiveness and motility in both scratch and Boyden chamber assays $(P<0.0005$; Supplementary Fig. S7 and [64]), therefore we examined system gains which might contribute to motility. Microtubule myosin components MYH10, MYL9, and MYLK together with associated proteins TPM1 and CALD1 were increased in the mesenchymal state, linking actin and intermediate filaments with myosin motors in a manner consistent with the acquisition of cell motility and characteristic of myofibroblasts. Decreased epithelial myosin MYO5C was observed. L-plastin is an actin filament protein expressed in fibroblast filapodia, where phosphorylation of S5 increases actin binding. Early in TGF $\beta$ mediated EMT phosphorylation of S5 and S257 are increased, which would activate and recruit L-plastin to an assembling actin cytoskeleton. S5 is a likely target of PKA [73]. Prominin-2, like prominin-1 (CD133) a cancer stem cell marker, appears to play a role in plasma membrane protrusions [74].
Phosphorylation of a serine core (S114-S118) in prominin-2 is increased early in TGF $\beta$ EMT. Taken together these data suggest a coupling of myosin, intermediate and actin filaments in EMT mediated motility.

Attenuation of autocrine EGFR family, Met family and IGF1R signaling networks with EMT

Our previous worked showed that EMT can promote resistance to EGFR and IGF1R tyrosine kinase inhibitors (TKIs) in multiple carcinoma types [27, 31]. Confirming these findings we observed: (1) EGFR, and IGF1R tyrosine phosphorylation was markedly attenuated in the mesenchymal state (Fig. 4a, b); (2) EGF family ligand expression was attenuated (Supplementary Table S5; [25]); (3) ErbB3, the preferred phosphatidyl inositol- $3^{\prime}$ kinase recruiting partner for EGFR and ErbB2, was transcriptionally silenced (Fig. 4a, Supplementary Table S5); (4) EGFR total protein abundance was unchanged regardless of EMT state (Supplementary Table S5). As mesenchymal cells are insensitive to EGFR inhibition we asked whether EGFR function was impaired in the mesenchymal state. EGF stimulated SHC and CBL tyrosine phosphorylation was inhibited by selective pharmacological EGFR inhibition in both epithelial and fixed-state mesenchymal lines (Fig. 4c), while a phosphorylation control HSPD1 was unaffected. These data indicate EGFR is functional and can respond to exogenous ligand in both epithelial and mesenchymal states, but is only autocrine activated in the epithelial state, contributing to EGFR onco-addiction in cells with an epithelial phenotype.

This extensive dataset allowed us to gain further insight into the lack of EGFR dependency in the mesenchymal like state. For example diverse regulators of EGFR function such as NKD2, required for export of TGF $\alpha$ to the cell surface subsequent to proteolytic release and EGFR binding [71], were decreased during EMT. Similarly LRIG, which directly interacts with and negatively regulates EGFR signaling [75] and receptor-type tyrosine-protein phosphatase kappa (PTPRK) that dephosphorylates and inactivates EGFR signaling [76] were both increased. Together with the observed attenuation of EGF-family ligand synthesis, these changes in direct EGFR regulators would further reduce EGFR activity in the mesenchymal state.

Interestingly, both Met and Ron receptor tyrosine kinases, which bind the ligands HGF and MSP, respectively and are associated with increased cell migration and metastasis, were decreased in expression and phosphotyrosine content in cells with mesenchymal-like phenotype (Fig. 4a; Supplementary Table S5). The attenuation of phosphotyrosine-dependent signaling through Met, Ron, EGFR, ErbB2, ErbB3 and IGF1R were confirmed by blot e.l.i.s.a. and quantitation of the chemi-luminescent signal $(P<0.01$; Fig. 4 b) . Downstream signaling nodes were 


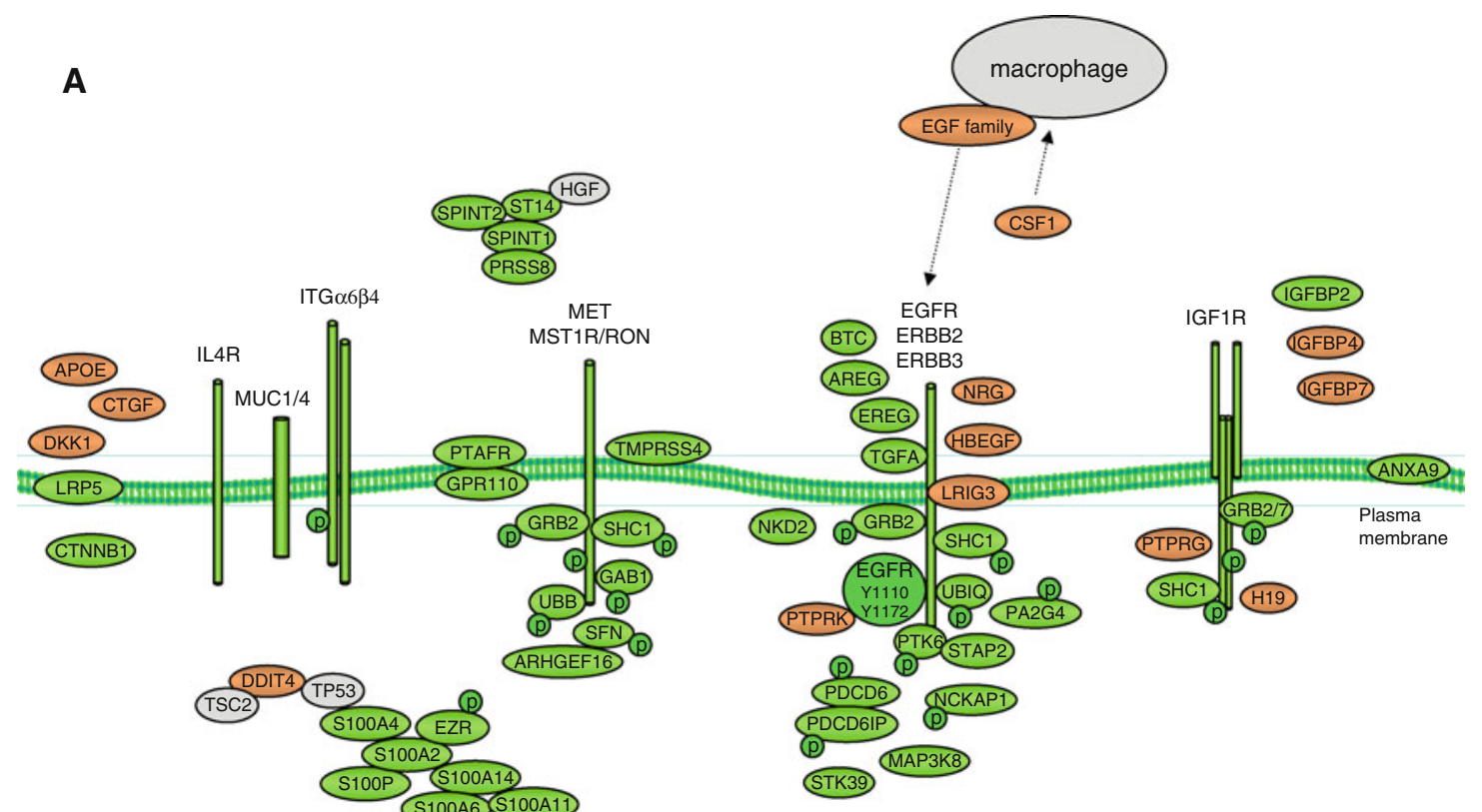

B

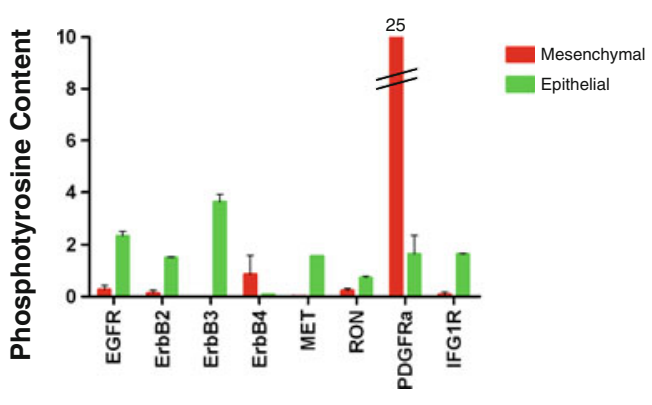

RTK tyrosine phosphorylation measured by elisa array, comparing ratios of mesenchymal-like (Mes) and epithelial (Epi) NSCLC lines: all changes $p<0.01$ except ErbB4 (ns) where error bars reflect the standard error of the mean

Fig. 4 a Loss of autocrine RTK signaling networks through EGFR/ ErbB2/ErbB3, Met/Ron and IGF1R signaling networks. Attenuated IL4R, Muc1 and Muc4 and integrin $\alpha 6 \beta 4$ components also were observed. b Attenuated RTK tyrosine phosphorylation in $\mathrm{H} 292$ and H358, (epithelial phenotype) Calu6 and H1703 (mesenchymal phenotype) cell models were confirmed by quantitation of RTK arrays. c EGFR is functional and responds to exogenous ligand in

co-attenuated with the EMT-mediated reduction in Met, Ron, EGFR and IGF1R kinase activation. These included multiple $\mathrm{SH} 2$, cytoplasmic kinase and ubiquinylation adapters: pSHC, pGrb2, pGAB1, pPTK6 (Brk), pUBB, pUBIQ and pNCKAP1. We asked whether attenuated Met

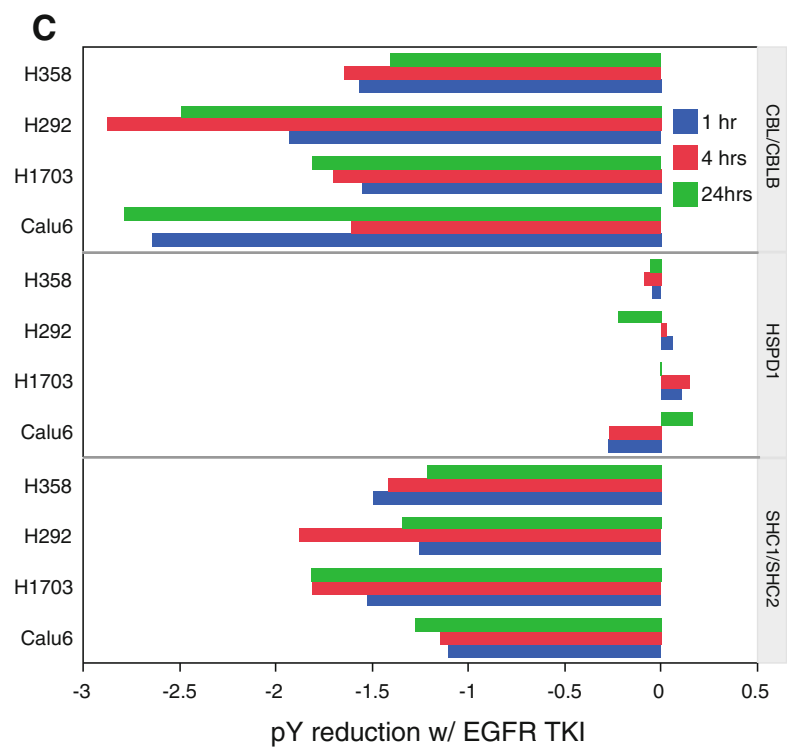

both epithelial and mesenchymal states. H292 and H358, (epithelial) Calu6 and H1703 (mesenchymal) cells were exposed to EGFR kinase inhibitor ( $3 \mu \mathrm{M}$ erlotinib, $2 \mathrm{~h}$ ) or DMSO control prior to stimulation with exogenous EGF $(10 \mathrm{ng} / \mathrm{ml}, 10 \mathrm{~min})$. The EGFR-dependent inhibition of substrates CBL and SHC were similar in epithelial and mesenchymal-like cells, relative for control HSPD1

phosphorylation might be attributable to reduced HGF binding to Met. While no change in HGF transcript abundance was observed, ST14 (matriptase), an extracellular protease which promotes proHGF activation, was markedly reduced. SPINT2 and SPINT1, which augment ST14 
activity, also were attenuated. Paradoxically while EGFR family, IGF1R and Met signaling can promote EMT, once transitions occur, signaling from these RTKs is attenuated. These data suggest that, like EGFR and IGF1R, Met and Ron play roles early in invasion and migration and are not required for maintenance of a mesenchymal-like cell state in multiple NSCLC models.

These observations raise the question as to why mesenchymal-like tumor cells simultaneously reduce signaling through EGFR family, IGF1R and Met RTK networks, normally important factors for epithelial tissue maintenance. We have shown that the EGFR signaling apparatus is still intact and can respond to exogenous EGF. A corollary of these data is that, in vivo, paracrine activation of EGFR, and possibly IGF1R and Met kinase activities are still enabled. We propose that autocrine to paracrine switching allows for chemotactic sensing of growth factor and cytokine gradients important to invasion. Evidence for this has been presented in a number of published studies. For example EMT-derived cells can migrate away from the tumor mass by chemotactic signaling with recruited macrophages [77]. Tumor cells provide paracrine CSF1 while recruited tumor associated macrophages secrete EGF family ligands, whose gradient allows migrational direction [10]. In this case tumor cells with autocrine EGFR signaling would be incapable of EGF-mediated directional chemotaxis, a deficit alleviated by EMT-like transition.

Another question that arises from these findings is what is promoting cell survival and preventing anoikis or senescence, in the absence of EGFR family, IGF1R, Met and Ron signaling, important survival networks for normal epithelium? We focused on acquired mesenchymal signaling through distinct networks not observed in epithelial backgrounds.

\section{EMT acquired survival networks}

Six major clusters indicating gains of autocrine pathway activation in mesenchymal-like NSCLC models were observed (Fig. 5a): IL11 and IL6 mediated gp130-JAK activation; Gas6 mediated Axl and Tyro3 activation; fibronectin-mediated $\alpha 5 \beta 1$-integrin activation; TGF $\beta$ - and BMP1- mediated receptor signaling; and autocrine FGFR and PDGFR networks were acquired in an EMT dependent manner. In addition, multiple direct and indirect modulators of cell survival were altered by EMT state.

The cytokine cascades stemming from inflammation of the tumor micro-environment play a key role in cancer progression and EMT [7, 10, 77]. Among the pro-inflammatory cytokines unregulated during EMT in the described models, IL6 and IL11 were of particular interest. IL6 was observed to increase during EMT in the H358-Snail and Zeb1 models but not in response to TGF $\beta$ stimulation
(Supplementary Table S5). In contrast IL-11 was observed to increase in all three of the H358 based EMT models (Supplementary Table S5). These cytokines are ligands for a family of receptors which couple through IL6ST/gp130 to signal through the JAK/STAT3 pathway to induce gene expression programs. Of interest, we also observe small increases in the receptors for these cytokines IL6R and IL6ST (gp130) (Supplementary Table S5) in the Snail and Zeb1 models. The production of these cytokines and increased expression of their receptors may have implications for both autocrine and paracrine signaling mechanisms. Both IL6 and IL11 [78] have been shown to induce pro-migratory and pro-invasive phenotypes on cancer cells and so it can be hypothesized that the increased expression of these cytokines during EMT can contribute to the invasive nature of mesenchymal-like tumor cells [79]. In addition, as both of these cytokines can activate the JAK/ STAT signaling pathways it provides a potential mechanistic explanation for the observed resistance of mesenchymal-like tumor cells to chemotherapeutic and molecular targeted therapies. Interestingly, increased IL-6 dependent signaling has recently been reported to provide resistance to EGFR targeted therapy [80] with the IL-6 production being linked to TGF $\beta$-mediated EMT [81]. Taken together these observations suggest that up regulation of IL6 and IL11 production in cancer cells as they undergo EMT is linked to a pro-metastatic, tumor progressive phenotype. A further understanding of the effects of these cytokines on tumor cells and the tumor microenvironment will lead to the identification of potential routes of therapeutic intervention. These data suggest dual EGFR/JAK-STAT targeting would be required for optimal therapeutic outcome in metastatic NSCLC with autocrine IL6ST-JAK-STAT signaling.

The production of these cytokines can also influence the tumor microenvironment in a paracrine fashion leading to enhanced tumor progression. IL6 and IL11 can influence the biology of lymphocytes, macrophages, hematopoeitic cells, endothelial cells and osteoclasts [82]. Of particular interest is the impact on osteoclasts, whereby IL11 signaling can induce bone resorption leading to a metastatic niche within bone amenable for tumor cell growth. Indeed, breast cancers expressing high levels of IL11 show an enhanced level of bone metastasis compared to those patients whose tumors express lower levels of IL11 [83]. Multiple cytokine regulators were altered with EMT state, including SIGIRR (TIR8), an IL1R/Toll-like receptor family member, functions as a negative regulator of inflammation [84]. SIGIRR was down regulated in all three H358 EMT models can contribute to the aberrant increase inflammatory cytokines in the mesenchymal state. Urocortin 2 (UCR2), a hypoxia inducible ligand for the corticotrophin receptor with antiangiogenic properties [85] was increased in metastable 
Fig. 5 a Gain of new survival and cytokine networks. Increased signaling through IL-11/IL-6/gp130, TGF $\beta$ / BMP2, Ax1/Tyro3, integrin $\alpha 5 \beta 1$, PDGFR and FGFR were observed. Downstream activation of BRAF/MAPK1 and PTK2/paxillin signaling were observed. Components in green were attenuated during EMT while those in light brown/red were increased with EMT. b Activating MAPK1/Erk phosphorylation (T185/Y187), associated with TGF $\beta$-induced EMT, was confirmed by immunoblot

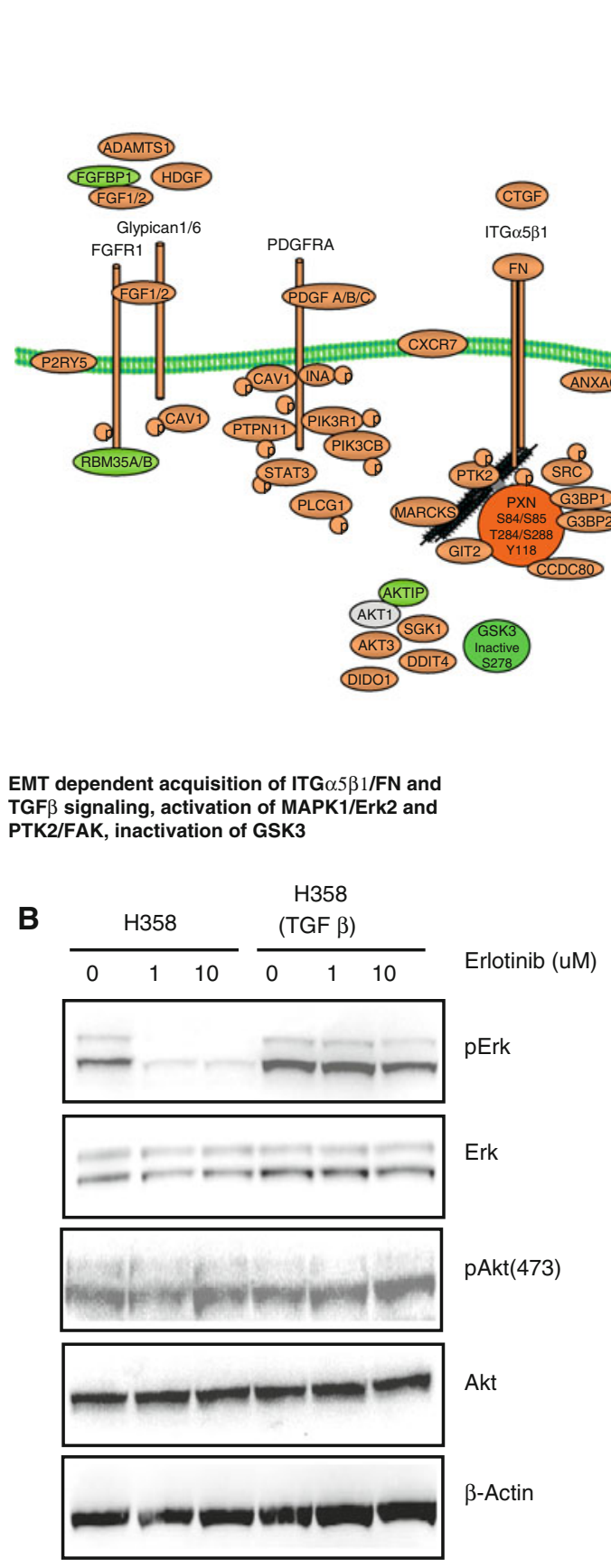

EMT. Opposing this function, multiple pro-angiogenic proteins were increased, notably angiopoietin-1 (ANGPT1), co-expression of thrombospondins together with the ADAMTS1 protease [86] and VEGF-C.

Multiple receptor protein kinase networks were activated in the mesenchymal state. Autocrine Gas6 stimulation of Axl and Tyro3 activity were established from array and RT-PCR RNA abundance measurements. The data suggest inhibitors of Axl and Tyro3 activity may show utility in limiting the survival of shed mesenchymal-like NSCLC cells. A functional role of autocrine Axl-Gas6 expression in lung [87] and breast cancer [88] progression has been established. Autocrine activation of FGFR and PDGFR signaling also was observed in both established (H1703) and metastable TGF $\beta$ models, supporting previous findings [25, 89]. This increase in kinase activity and PDGFR autophosphorylation was associated with increased PDGFR and PDGF RNA transcripts. In metastable Snail and Zeb1 models, FGFR1 and PDGFR transcripts were markedly increased, PDGF was modestly increased and FGF1 was unchanged or slightly decreased (Supplementary Table S5). These data support the prediction of kinase activation by autocrine co-expression of receptor-ligand transcripts. We asked what functional 
consequence PDGFR and FGFR exerted in the multiple model systems. While pharmacological inhibition of PDGFR or FGFR elicited a marked decrease in H1703 proliferation [25, 89], inhibition of PDGFR or FGFR had little or no activity in the metastable models, despite initiation of autocrine activation (data not shown). These data suggest cellular onco-addiction to active PDGFR and FGFR networks is a trait selected for relatively late following EMT and that PDGFR and FGFR networks are not required for either the EMT transition itself or for immediate survival following EMT. The activation of TGF $\beta$ R signaling is a physiologically relevant and frequent promoter of EMT in many model systems. Increased transcription of TGF $\beta 1,2$ and 3 as well as increased levels of TGF $\beta$ receptors 1,2 and BMP receptor 2 were observed. This was associated with activating phosphorylation of Erk but not Akt (Fig. 5a, b). However both Snail and Zeb1 metastable models were insensitive to $\mathrm{TGF} \beta \mathrm{R}$ inhibition (data not shown), again suggesting cellular dependence on TGF $\beta$ signaling may be a trait selected for relatively late following EMT or restricted to TGF $\beta$-initiated EMT.

As described earlier, H358/TGF $\beta$ cells markedly upregulated expression of the fibronectin receptor integrin $\alpha 5 \beta 1$ together with autocrine fibronectin expression. Autocrine integrin $\alpha 5 \beta 1$ activation was also observed in the committed mesenchymal-like lines H460 (data not shown) and subclones of the H292 line (data not shown) supporting its prominence in both metastable and fixed mesenchymal states. In the H358/ TGF $\beta$ model autocrine integrin activation accompanied tyrosine phosphorylation of PTK2 (FAK), paxillin, BCAR1 (p130CAS) linking this complex to the filamin A, filamin B components of the focal adhesion-actin cytoskeleton. The BCAR binding adapter protein SH2D3A, a component of the PTK2/paxillin/actin complex, showed increased phosphorylation on $\mathrm{S} 125$ in the metastable TGF $\beta$ model.

Resistance to chemotherapy-induced apoptosis is a characteristic of mesenchymal-like tumors and lines [21, 22]. Prohibitins-1 and -2 were increased with EMT (Supplementary Table S5) and have been shown to displace 14-3-3 from raf to allow ras localization and activation [90]. In this regard, increased Erk phosphorylation on the activation loop was common in both the metastable H358 (Fig. 5b) and fixed state models. Both Erk/2MAPK1 and p38/MAPK14 showed increased tyrosine phosphorylation in the H358/TGF $\beta$ model (Supplementary Table S5), consistent with increased phosphorylation of Y187 for MAPK1 (Supplementary Table S9) and enzyme activation. The down regulation of PKN1 (in all H358 models) and subsequent reduction in the phosphorylation of TRAF2 may relieve negative control over both NFkB and p38/JNK pathways. Activation of p38 $\alpha$ /MAPK14 and p38IP have been shown to be required for EMT during mouse gastrulation [91]. While cross correlation of individual protein and gene changes between fractions and experiments increases the confidence in quantitation, the combined measurement of system components unequivocally establish reorganization of receptor protein kinase and integrin complexes during EMT.

EMT-associated transcription nodes and alternative splicing

We asked whether subsets of changes observed in mesenchymal-like phenotypes could be correlated with specific transcription components. We used pathway analysis to build networks in two ways. Firstly, we built out from EMT modulated transcription factors to modulated genes and in a second complementary approach in from EMT modulated genes back to proposed transcription nodes. The identified EMT transcription nodes included NFkB2/RelA, ATF3/ ATF2, Myc, FOXA1/FOXA2, ETS1, NFE2L2/L3, MEF2C, Snail/Slug [45, 92] and Zeb1/Zeb2 [93, 94]. These networks are outlined in Fig. 6a, b. The loss of FOXA1 and FOXA2 was recently been shown to result in the loss of E-cadherin (CDH1) and were required for EMT in pancreas cancer cells in vitro [95]. Snail is reported to be a critical regulator of EMT and was readily identified as a node in our analysis. The levels of Snail itself were increased in the EMT models, as were factors reported which influence its stability and activity. The serine-threonine kinases PAK1, PAK2 and the lysyl oxidases (LOX, LOXL1 and LOXL2), which participate to enhance Snail stability [96], were increased in mesenchymal-like NSCLC cells. The dephosphorylation $(-2.2$ $\log 2$ ) of GSK $3 \alpha / \beta$ on the activating site Y279 also may contribute to PAK activation and Snail stabilization associated with EMT. Supporting this hypothesis, phosphorylation of PAK2 on S141, an activating phosphorylation site [97], appeared increased in TGF $\beta$ mediated EMT $(1.5 \log 2)$ and increased Snail transcripts were observed in TGF $\beta$ and Zeb1 models. Finally HDAC7 cytoplasmic-nuclear localization is regulated by phosphorylation on $\mathrm{S} 486$ by PKD or CAMK and recruitment of 14-3-3 proteins. In TGF $\beta$ induced EMT this site is hypophosphorylated, which would lead to nuclear localization and repression of target genes.

In addition to transcriptional reprogramming it is clear that mRNA splicing can change the repertoire of proteins synthesized during EMT. Published examples of this include the alternative splicing of FGFR and ENAH (Mena) that generate proteins with different functional consequences in epithelial compared to mesenchymal cells [46]. A relatively large cluster of 24 proteins associated with RNA maturation, alternative splicing and RNA export were increased, contributing to post-transcriptional RNA processing associated with the mesenchymal phenotype and may be functionally linked to focal adhesion complex changes [98]. In contrast, mesenchymal cells demonstrated a marked decrease in the 
Fig. 6 a The transcription node regulating interferon response was modulated during EMT. a Reduced free radical scavenging capacity was correlated the mesenchymal state, associated with the NFE2L2 transcription node. Components in green were attenuated during EMT while those in light brown/red were increased with EMT.

b Networks regulated during EMT associated with FOXA, Snail, Zeb, ATF, NFKB/Rel, MEF2and Myc transcription nodes
A
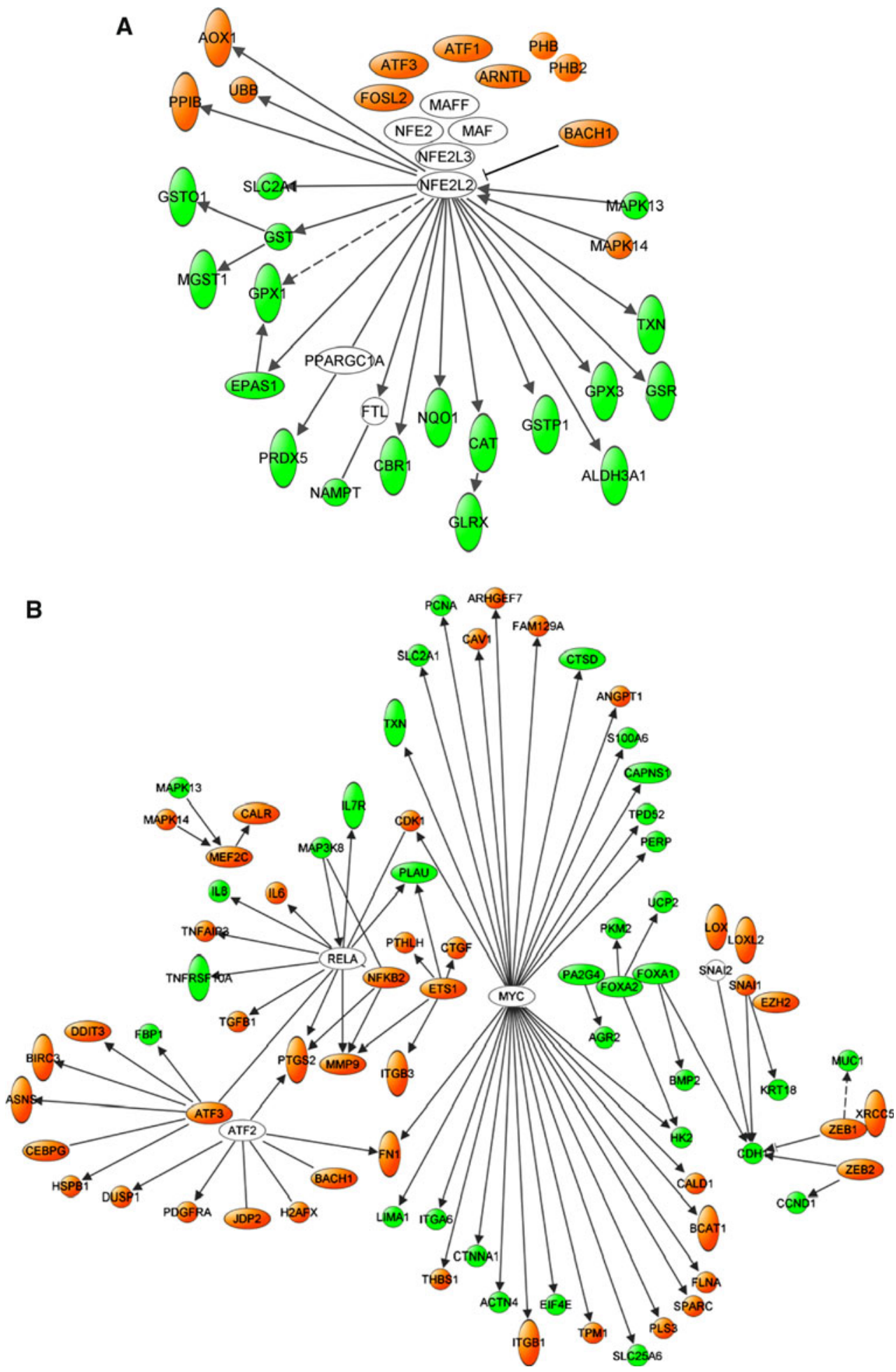

splicing factors RBM35A (ESRP1), RBM35B (ESRP2), RBM47, STAU2 and STRBP. Of note, RBM35A and RBM35B are required for the epithelial splice form of FGFR1 and FGFR2, known to be alternatively spliced between EMT states [46] and where the mesenchymal splice form of FGFR shows gain of function.
Cell stress and metabolism

The previous sections have indicated that during metastable EMT, cells have exchanged proliferative potential for migratory capability. We asked what metabolic changes might be associated with this reduction in replicative stress 
and the reduced need for macromolecular biosynthesis. Three functional metabolic groups were modulated by EMT: (1) the redox-stress pathway was markedly reduced in capacity; (2) the glycolytic and oxidative phosphorylation pathways appeared down and up regulated, respectively; and (3) the prostaglandin/prostacyclin pathway was upregulated.

Altered metabolism of cancer cells often leads to the excess production of reactive oxygen species (ROS) which are scavenged in part by the glutathione and thioredoxin pathways [99]. Induction of ROS scavenger pathways has been associated with resistance to chemotherapeutic agents which increase free radical production. As mesenchymal tumor cells show relative resistance to multiple classes of chemotherapy, the consistent reduction in the abundance of seventeen members of the ROS scavenger pathway was unexpected (Fig. 6a). Notably glutathione S-transferase (GSTP1/O1), perioredoxin (PRDX2/5) and thioredoxin (TXN) were all attenuated. ROS stress sensing is regulated in part by phosphorylation and nuclear import/export of the Cap'n' collar proteins NFE2L2/L3 which bind to antioxidant response elements within target genes. DNA binding is modulated by multiple proteins upregulated by EMT (FOSL2, ATF3, ARNTL, PHB and PHB2) [100]. NFE2L2 family activation of anti-oxidant genes is negatively regulated by the dominant negative Cap'n'collar protein BACH1. BACH1 was increased with metastable EMT and would block NFE2L2/L3-mediated increases in redox stress gene expression. Glutathione synthesis genes also can be regulated by the Myc transcription factor. The decreased abundance of redox stress pathway components indicate reduced activation of NFE2L2/L3 transcription and/or Myc pathways in both metastable and fixed state models. This finding is of interest since redox stress can promote EMTlike transitions [101], but once EMT has occurred, the activation of free radical induced genes do not appear critical to a maintenance of the mesenchymal state.

Intersecting DNA damage cellular stress pathways were similarly decreased with EMT. Histone H2AX, a marker of DNA repair, is phosphorylated on both S139 and Y142. The dephosphorylation of Y142 is associated with response to DNA damage $[102,103]$. In both fixed and metastable models $\mathrm{H} 2 \mathrm{AX}$ and $\mathrm{H} 2 \mathrm{AZ}$ showed increased phosphotyrosine content in the mesenchymal state, indicative of a reduction in the sensing of DNA damage. While GADD $45 \beta$ was up regulated in the three metastable models, taken together the data suggest the GADD $45 \beta$ increase may reflect cell cycle delay rather than DNA damage response. A coordinate reduction in DNA damage response and redox stress pathway components was associated with a mesenchymal-like cell state.

The shift from oxidative phosphorylation to aerobic glycolysis and increased biosynthesis is a hallmark of cancer [104]. In turn oxidative stress leads to an excess in ROS production and an up regulation of glutathione/thioredoxin scavenger capacity. Through EMT a decrease in ROS inducible genes was observed. We also observed a reduction in the abundance of the glycolytic HK2, PFK, FBP1, PKM2, ME1, GLUD1 and in glutamate/non-essential amino acid transporters SLC1A1 and the CD98/4F2 complex (SLC7A5 and SLC3A2), indicative of reduced glycolytic capacity and reduced oxidative stress. In contrast, mitochondrial complex I, III and V components NADH dehydrogenase (NDUA9), Ubiquinol-cytochrome $\mathrm{c}$ reductase (UCRC) and ATP synthase subunits $5 \mathrm{~B} / 5 \mathrm{C} 1 / 5 \mathrm{D}$ were increased with transition to a more mesenchymal-like phenotype. Seven members of the amino acid metabolism/urea cycle pathway were coordinately decreased during EMT, including CKB, GATM, GLUD1 and GLUD2, OAT, PRODH, and SAT1. EMT also resulted in a reduction in transcripts encoding fatty acid synthase (FASN) and fatty acid hydroxylase (FA2H), suggesting reduced synthesis of palmitate and hydroxy-spingolipids. Taken together these data suggest EMT shifts cellular metabolism to reduce glycolytic capacity, reduce oxidative stress and limit fatty acid synthesis.

Prostaglandin/prostacyclin metabolism was modulated by EMT state in a manner consistent with increased prostaglandin $\mathrm{H} 2$ and prostacyclin availability. The cyclooxygenase PTGS2 (COX-2) was increased while carbonyl reductase was decreased, which together would increase the abundance of the pro-inflammatory cytokine PGE2. However offsetting decreases in prostaglandin E synthases1 and -3 would increase prostaglandin $\mathrm{H} 2$ and prostacyclin abundance. An inflammatory microenvironment and elevated COX-2 have been correlated with increased EMTlike transitions $[9,105]$.

\section{Conclusions}

Here we have developed and extensively analysed a number of models of the EMT process that reflect multiple different stages of the transition. It is clear from these studies that analsyes of a single model system by a single methodology give only a limited insight into the EMT process as a whole. For this reason we undertook a more extensive systems biology approach to analyze both inducible models of EMT in an isogenic background as well as epigenetically fixed cancer cell lines of both epithelial and mesenchymal phenotypes. From these analyses it is clear that there are a number of recurring common themes in the types of transcriptional and protein regulatory changes that are observed during EMT. These include marked changes in the repertoire of cell-cell junctional proteins, increases in pro-invasive and pro-migratory proteins, changes in the flux through metabolic pathways and a change in the repertoire of secreted cytokines that modify 
the tumor microenvironment. All of these changes are controlled through the actions of a number of critical transcription factors and the networks of factors that they induce.

Epithelial mesenchymal transitions resulted in marked changes to cellular survival signaling and metabolic networks. A shift from EGFR, IGF1R and Met/Ron activated signaling networks to a new repertoire of autocrine survival networks was clear. These included autocrine IL11/IL6gp130/JAK2/STAT, fibronectin-integrin, GAS6-Ax1/Tyro3, PDGFR/FGFR/RET and TGF $\beta$ R networks, often in a heterogeneous cell-specific manner. Preliminary evidence suggest a model where initial dependence on IL11/IL6JAK/STAT, TGF $\beta$ R and/or fibronectin-integrin signaling in the early metastable state, gives way to later cell selection and onco-addiction to Axl, Tyro3, PDGFR, FGFR or RET networks in the epigenetically-fixed sarcoma-like state where tumor cells can both proliferate and migrate. The reductions in oxidative and redox stress networks with EMT were unexpected and suggest new strategies may be required to effectively exploit cancer metabolism in epithelial, metastable and fixed-mesenchymal states. The measured changes in survival and metabolic networks provide new opportunities to pharmacologically target both epithelial and mesenchymal tumor cell states, important to reduce cancer progression and recurrence. These network changes can be functionally elucidated by systematic large scale shRNAi screening for EMT phenotype and mesenchymal cell survival.

Open Access This article is distributed under the terms of the Creative Commons Attribution Noncommercial License which permits any noncommercial use, distribution, and reproduction in any medium, provided the original author(s) and source are credited.

\section{References}

1. Thiery JP et al (2009) Epithelial-mesenchymal transitions in development and disease. Cell 139(5):871-890

2. Yang J, Weinberg RA (2008) Epithelial-mesenchymal transition: at the crossroads of development and tumor metastasis. Dev Cell 14(6):818-829

3. Hill $\mathrm{R}$ et al (2005) Selective evolution of stromal mesenchyme with p53 loss in response to epithelial tumorigenesis. Cell 123(6):1001-1011

4. Zeisberg EM et al (2007) Discovery of endothelial to mesenchymal transition as a source for carcinoma-associated fibroblasts. Cancer Res 67(21):10123-10128

5. Orimo A et al (2005) Stromal fibroblasts present in invasive human breast carcinomas promote tumor growth and angiogenesis through elevated SDF-1/CXCL12 secretion. Cell 121(3):335-348

6. Gupta GP, Massague J (2006) Cancer metastasis: building a framework. Cell 127(4):679-695

7. Tlsty TD, Coussens LM (2006) Tumor stroma and regulation of cancer development. Annu Rev Pathol 1:119-150
8. Kalluri R, Zeisberg M (2006) Fibroblasts in cancer. Nat Rev Cancer 6(5):392-401

9. Dohadwala M et al (2006) Cyclooxygenase-2-dependent regulation of E-cadherin: prostaglandin E(2) induces transcriptional repressors ZEB1 and snail in non-small cell lung cancer. Cancer Res 66(10):5338-5345

10. Condeelis J, Pollard JW (2006) Macrophages: obligate partners for tumor cell migration, invasion, and metastasis. Cell 124(2): 263-266

11. Patsialou A et al (2009) Invasion of human breast cancer cells in vivo requires both paracrine and autocrine loops involving the colony-stimulating factor-1 receptor. Cancer Res 69(24): 9498-9506

12. Vega $\mathrm{S}$ et al (2004) Snail blocks the cell cycle and confers resistance to cell death. Genes Dev 18(10):1131-1143

13. Grunert S, Jechlinger M, Beug H (2003) Diverse cellular and molecular mechanisms contribute to epithelial plasticity and metastasis. Nat Rev Mol Cell Biol 4(8):657-665

14. Yamaguchi H, Wyckoff J, Condeelis J (2005) Cell migration in tumors. Curr Opin Cell Biol 17(5):559-564

15. Prasad M, Montell DJ (2007) Cellular and molecular mechanisms of border cell migration analyzed using time-lapse livecell imaging. Dev Cell 12(6):997-1005

16. Thiery JP (2002) Epithelial-mesenchymal transitions in tumour progression. Nat Rev Cancer 2(6):442-454

17. Tsuji T, Ibaragi S, Hu GF (2009) Epithelial-mesenchymal transition and cell cooperativity in metastasis. Cancer Res 69(18):7135-7139

18. Damonte $P$ et al (2007) EMT tumorigenesis in the mouse mammary gland. Lab Invest 87(12):1218-1226

19. Blaukovitsch M et al (2006) Sarcomatoid carcinomas of the lung-are these histogenetically heterogeneous tumors? Virchows Arch 449(4):455-461

20. Mani SA et al (2008) The epithelial-mesenchymal transition generates cells with properties of stem cells. Cell 133(4): 704-715

21. Arumugam T et al (2009) Epithelial to mesenchymal transition contributes to drug resistance in pancreatic cancer. Cancer Res 69(14):5820-5828

22. Cheng GZ et al (2007) Twist transcriptionally up-regulates AKT2 in breast cancer cells leading to increased migration, invasion, and resistance to paclitaxel. Cancer Res 67(5):1979-1987

23. Ansieau $\mathrm{S}$ et al (2008) Induction of EMT by twist proteins as a collateral effect of tumor-promoting inactivation of premature senescence. Cancer Cell 14(1):79-89

24. Buck E et al (2008) Feedback mechanisms promote cooperativity for small molecule inhibitors of epidermal and insulin-like growth factor receptors. Cancer Res 68(20):8322-8332

25. Thomson $\mathrm{S}$ et al (2008) Kinase switching in mesenchymal-like non-small cell lung cancer lines contributes to EGFR inhibitor resistance through pathway redundancy. Clin Exp Metastasis 25(8):843-854

26. Adam L et al (2009) miR-200 expression regulates epithelial-tomesenchymal transition in bladder cancer cells and reverses resistance to epidermal growth factor receptor therapy. Clin Cancer Res 15(16):5060-5072

27. Buck E et al (2007) Loss of homotypic cell adhesion by epithelial-mesenchymal transition or mutation limits sensitivity to epidermal growth factor receptor inhibition. Mol Cancer Ther $6(2): 532-541$

28. Frederick BA et al (2007) Epithelial to mesenchymal transition predicts gefitinib resistance in cell lines of head and neck squamous cell carcinoma and non-small cell lung carcinoma. Mol Cancer Ther 6(6):1683-1691

29. Fuchs BC et al (2008) Epithelial-to-mesenchymal transition and integrin-linked kinase mediate sensitivity to epidermal growth 
factor receptor inhibition in human hepatoma cells. Cancer Res 68(7):2391-2399

30. Shrader M et al (2007) Molecular correlates of gefitinib responsiveness in human bladder cancer cells. Mol Cancer Ther 6(1):277-285

31. Thomson S et al (2005) Epithelial to mesenchymal transition is a determinant of sensitivity of non-small-cell lung carcinoma cell lines and xenografts to epidermal growth factor receptor inhibition. Cancer Res 65(20):9455-9462

32. Witta SE et al (2006) Restoring E-cadherin expression increases sensitivity to epidermal growth factor receptor inhibitors in lung cancer cell lines. Cancer Res 66(2):944-950

33. Yauch RL et al (2005) Epithelial versus mesenchymal phenotype determines in vitro sensitivity and predicts clinical activity of erlotinib in lung cancer patients. Clin Cancer Res 11(24 Pt 1):8686-8698

34. Angelucci A et al (2006) Suppression of EGF-R signaling reduces the incidence of prostate cancer metastasis in nude mice. Endocr Relat Cancer 13(1):197-210

35. Lo HW et al (2007) Epidermal growth factor receptor cooperates with signal transducer and activator of transcription 3 to induce epithelial-mesenchymal transition in cancer cells via up-regulation of TWIST gene expression. Cancer Res 67(19): 9066-9076

36. Kim HJ et al (2007) Constitutively active type I insulin-like growth factor receptor causes transformation and xenograft growth of immortalized mammary epithelial cells and is accompanied by an epithelial-to-mesenchymal transition mediated by NF-kappaB and snail. Mol Cell Biol 27(8):3165-3175

37. Brabletz $\mathrm{T}$ et al (2005) Opinion: migrating cancer stem cellsan integrated concept of malignant tumour progression. Nat Rev Cancer 5(9):744-749

38. Moody SE et al (2005) The transcriptional repressor Snail promotes mammary tumor recurrence. Cancer Cell 8(3): 197-209

39. Gossen $M$ et al (1994) Inducible gene expression systems for higher eukaryotic cells. Curr Opin Biotechnol 5(5):516-520

40. Petti F et al (2005) Temporal quantitation of mutant Kit tyrosine kinase signaling attenuated by a novel thiophene kinase inhibitor OSI-930. Mol Cancer Ther 4(8):1186-1197

41. Thelemann A et al (2005) Phosphotyrosine signaling networks in epidermal growth factor receptor overexpressing squamous carcinoma cells. Mol Cell Proteomics 4(4):356-376

42. Ross PL et al (2004) Multiplexed protein quantitation in Saccharomyces cerevisiae using amine-reactive isobaric tagging reagents. Mol Cell Proteomics 3(12):1154-1169

43. Shilov IV et al (2007) The Paragon Algorithm, a next generation search engine that uses sequence temperature values and feature probabilities to identify peptides from tandem mass spectra. Mol Cell Proteomics 6(9):1638-1655

44. Bradshaw RA et al (2006) Reporting protein identification data: the next generation of guidelines. Mol Cell Proteomics 5(5): 787-788

45. Peinado H, Olmeda D, Cano A (2007) Snail, Zeb and bHLH factors in tumour progression: an alliance against the epithelial phenotype? Nat Rev Cancer 7(6):415-428

46. Oltean $\mathrm{S}$ et al (2006) Alternative inclusion of fibroblast growth factor receptor 2 exon IIIc in Dunning prostate tumors reveals unexpected epithelial mesenchymal plasticity. Proc Natl Acad Sci USA 103(38):14116-14121

47. Dumont $N$ et al (2008) Sustained induction of epithelial to mesenchymal transition activates DNA methylation of genes silenced in basal-like breast cancers. Proc Natl Acad Sci USA 105(39):14867-14872

48. Dennis G Jr et al (2003) DAVID: database for annotation, visualization, and integrated discovery. Genome Biol 4(5):P3
49. da Huang W, Sherman BT, Lempicki RA (2009) Systematic and integrative analysis of large gene lists using DAVID bioinformatics resources. Nat Protoc 4(1):44-57

50. Andarawewa KL et al (2007) Ionizing radiation predisposes nonmalignant human mammary epithelial cells to undergo transforming growth factor beta induced epithelial to mesenchymal transition. Cancer Res 67(18):8662-8670

51. Blick T et al (2010) Epithelial mesenchymal transition traits in human breast cancer cell lines parallel the CD44(hi/)CD24 (lo/-) stem cell phenotype in human breast cancer. J Mammary Gland Biol Neoplasia 15(2):235-252

52. Carro MS et al (2010) The transcriptional network for mesenchymal transformation of brain tumours. Nature 463(7279): 318-325

53. Choi YL et al (2010) LYN is a mediator of epithelial-mesenchymal transition and a target of dasatinib in breast cancer. Cancer Res 70(6):2296-2306

54. Coldren CD et al (2006) Baseline gene expression predicts sensitivity to gefitinib in non-small cell lung cancer cell lines. Mol Cancer Res 4(8):521-528

55. Creighton CJ et al (2008) Development of resistance to targeted therapies transforms the clinically associated molecular profile subtype of breast tumor xenografts. Cancer Res 68(18): 7493-7501

56. De Craene B et al (2005) The transcription factor snail induces tumor cell invasion through modulation of the epithelial cell differentiation program. Cancer Res 65(14):6237-6244

57. Hennessy BT et al (2009) Characterization of a naturally occurring breast cancer subset enriched in epithelial-tomesenchymal transition and stem cell characteristics. Cancer Res 69(10):4116-4124

58. Jorissen RN et al (2009) Metastasis-associated gene expression changes predict poor outcomes in patients with dukes stage B and C colorectal cancer. Clin Cancer Res 15(24):7642-7651

59. Joyce $\mathrm{T}$ et al (2009) A molecular signature for Epithelial to Mesenchymal transition in a human colon cancer cell system is revealed by large-scale microarray analysis. Clin Exp Metastasis 26(6):569-587

60. Keshamouni VG et al (2009) Temporal quantitative proteomics by iTRAQ 2D-LC-MS/MS and corresponding mRNA expression analysis identify post-transcriptional modulation of actin-cytoskeleton regulators during TGF-beta-Induced epithelial-mesenchymal transition. J Proteome Res 8(1):35-47

61. Keshamouni VG et al (2006) Differential protein expression profiling by iTRAQ-2DLC-MS/MS of lung cancer cells undergoing epithelial-mesenchymal transition reveals a migratory/ invasive phenotype. J Proteome Res 5(5):1143-1154

62. Lien HC et al (2007) Molecular signatures of metaplastic carcinoma of the breast by large-scale transcriptional profiling: identification of genes potentially related to epithelial-mesenchymal transition. Oncogene 26(57):7859-7871

63. Lim E et al (2010) Transcriptome analyses of mouse and human mammary cell subpopulations reveal multiple conserved genes and pathways. Breast Cancer Res 12(2):R21

64. Argast GM et al (2010) Cooperative signaling between oncostatin $\mathrm{M}$, hepatocyte growth factor and transforming growth factor-beta enhances epithelial to mesenchymal transition in lung and pancreatic tumor models. Cells Tissues Organs 193(1-2):114-132

65. Vijayakumar S et al (2009) Phosphorylation of human DNA ligase I regulates its interaction with replication factor $\mathrm{C}$ and its participation in DNA replication and DNA repair. Mol Cell Biol 29(8):2042-2052

66. Birchmeier W, Weidner KM, Behrens J (1993) Molecular mechanisms leading to loss of differentiation and gain of invasiveness in epithelial cells. J Cell Sci Suppl 17:159-164 
67. Moreno-Bueno G, Portillo F, Cano A (2008) Transcriptional regulation of cell polarity in EMT and cancer. Oncogene 27(55):6958-6969

68. Singh AB, Sharma A, Dhawan P (2010) Claudin family of proteins and cancer: an overview. J Oncol 541957

69. Harris TJ, Tepass U (2010) Adherens junctions: from molecules to morphogenesis. Nat Rev Mol Cell Biol 11(7):502-514

70. Yin T, Green KJ (2004) Regulation of desmosome assembly and adhesion. Semin Cell Dev Biol 15(6):665-677

71. Li C et al (2004) Myristoylated Naked2 escorts transforming growth factor alpha to the basolateral plasma membrane of polarized epithelial cells. Proc Natl Acad Sci USA 101(15): $5571-5576$

72. Maeda M, Johnson KR, Wheelock MJ (2005) Cadherin switching: essential for behavioral but not morphological changes during an epithelium-to-mesenchyme transition. J Cell Sci 118(Pt 5):873-887

73. Janji B et al (2006) Phosphorylation on Ser5 increases the F-actin-binding activity of L-plastin and promotes its targeting to sites of actin assembly in cells. J Cell Sci 119(Pt 9):1947-1960

74. Florek $\mathrm{M}$ et al (2007) Prominin-2 is a cholesterol-binding protein associated with apical and basolateral plasmalemmal protrusions in polarized epithelial cells and released into urine. Cell Tissue Res 328(1):31-47

75. Rubin C, Gur G, Yarden Y (2005) Negative regulation of receptor tyrosine kinases: unexpected links to c-Cbl and receptor ubiquitylation. Cell Res 15(1):66-71

76. Xu Y et al (2006) Oxidative inhibition of receptor-type proteintyrosine phosphatase kappa by ultraviolet irradiation activates epidermal growth factor receptor in human keratinocytes. J Biol Chem 281(37):27389-27397

77. Qian BZ, Pollard JW (2010) Macrophage diversity enhances tumor progression and metastasis. Cell 141(1):39-51

78. Suman $P$ et al (2009) Interleukin-11 increases invasiveness of JEG-3 choriocarcinoma cells by modulating STAT3 expression. J Reprod Immunol 82(1):1-11

79. Sullivan NJ et al (2009) Interleukin-6 induces an epithelialmesenchymal transition phenotype in human breast cancer cells. Oncogene 28(33):2940-2947

80. Gao SP et al (2007) Mutations in the EGFR kinase domain mediate STAT3 activation via IL-6 production in human lung adenocarcinomas. J Clin Invest 117(12):3846-3856

81. Yao Z et al (2010) TGF-beta IL-6 axis mediates selective and adaptive mechanisms of resistance to molecular targeted therapy in lung cancer. Proc Natl Acad Sci USA 107(35):15535-15540

82. Putoczki T, Ernst M (2010) More than a sidekick: the IL-6 family cytokine IL-11 links inflammation to cancer. J Leukoc Biol 88(6):1109-1117

83. Sotiriou $C$ et al (2001) Interleukins-6 and -11 expression in primary breast cancer and subsequent development of bone metastases. Cancer Lett 169(1):87-95

84. Garlanda C et al (2007) Increased susceptibility to colitisassociated cancer of mice lacking TIR8, an inhibitory member of the interleukin-1 receptor family. Cancer Res 67(13): $6017-6021$

85. Hao $\mathrm{Z}$ et al (2008) Urocortin2 inhibits tumor growth via effects on vascularization and cell proliferation. Proc Natl Acad Sci USA 105(10):3939-3944

86. Lee YJ et al (2010) Variable inhibition of thrombospondin 1 against liver and lung metastases through differential activation of metalloproteinase ADAMTS1. Cancer Res 70(3):948-956
87. Li Y et al (2009) Axl as a potential therapeutic target in cancer: role of Axl in tumor growth, metastasis and angiogenesis. Oncogene 28(39):3442-3455

88. Gjerdrum C et al (2010) Axl is an essential epithelial-tomesenchymal transition-induced regulator of breast cancer metastasis and patient survival. Proc Natl Acad Sci USA 107(3):1124-1129

89. Rikova K et al (2007) Global survey of phosphotyrosine signaling identifies oncogenic kinases in lung cancer. Cell 131(6):1190-1203

90. Rajalingam K et al (2005) Prohibitin is required for Ras-induced Raf-MEK-ERK activation and epithelial cell migration. Nat Cell Biol 7(8):837-843

91. Zohn IE et al (2006) p38 and a p38-interacting protein are critical for downregulation of E-cadherin during mouse gastrulation. Cell 125(5):957-969

92. Batlle E et al (2000) The transcription factor snail is a repressor of E-cadherin gene expression in epithelial tumour cells. Nat Cell Biol 2(2):84-89

93. Aigner K et al (2007) The transcription factor ZEB1 (deltaEF1) promotes tumour cell dedifferentiation by repressing master regulators of epithelial polarity. Oncogene 26(49): 6979-6988

94. Spaderna $\mathrm{S}$ et al (2008) The transcriptional repressor ZEB1 promotes metastasis and loss of cell polarity in cancer. Cancer Res 68(2):537-544

95. Song Y, Washington MK, Crawford HC (2010) Loss of FOXA1/ 2 is essential for the epithelial-to-mesenchymal transition in pancreatic cancer. Cancer Res 70(5):2115-2125

96. Yang $\mathrm{Z}$ et al (2005) Pak1 phosphorylation of snail, a master regulator of epithelial-to-mesenchyme transition, modulates snail's subcellular localization and functions. Cancer Res 65(8):3179-3184

97. Chong C et al (2001) The mechanism of PAK activation. Autophosphorylation events in both regulatory and kinase domains control activity. J Biol Chem 276(20):17347-17353

98. de Hoog CL, Foster LJ, Mann M (2004) RNA and RNA binding proteins participate in early stages of cell spreading through spreading initiation centers. Cell 117(5):649-662

99. Trachootham D, Alexandre J, Huang P (2009) Targeting cancer cells by ROS-mediated mechanisms: a radical therapeutic approach? Nat Rev Drug Discov 8(7):579-591

100. Sykiotis GP, Bohmann D (2010) Stress-activated cap'n'collar transcription factors in aging and human disease. Sci Signal 3(112):re3

101. Radisky DC et al (2005) Rac1b and reactive oxygen species mediate MMP-3-induced EMT and genomic instability. Nature 436(7047):123-127

102. Cook PJ et al (2009) Tyrosine dephosphorylation of H2AX modulates apoptosis and survival decisions. Nature 458(7238): 591-596

103. Xiao A et al (2009) WSTF regulates the H2A.X DNA damage response via a novel tyrosine kinase activity. Nature 457(7225): $57-62$

104. Vander Heiden MG, Cantley LC, Thompson CB (2009) Understanding the Warburg effect: the metabolic requirements of cell proliferation. Science 324(5930):1029-1033

105. St John MA et al (2009) Proinflammatory mediators upregulate snail in head and neck squamous cell carcinoma. Clin Cancer Res 15(19):6018-6027 